\title{
Endoplasmic Reticulum Stress Is Important for the Manifestations of $\alpha$-Synucleinopathy In Vivo
}

\author{
Emanuela Colla, ${ }^{1}$ Philippe Coune, ${ }^{2}$ Ying Liu, ${ }^{1}$ Olga Pletnikova, ${ }^{1}$ Juan C Troncoso, ${ }^{1}$ Takeshi Iwatsubo, ${ }^{3}$ \\ Bernard L. Schneider, ${ }^{2}$ and Michael K. Lee ${ }^{1,4,5}$ \\ ${ }^{1}$ Department of Pathology, Johns Hopkins University School of Medicine, Baltimore, Maryland 21205, ${ }^{2}$ Brain Mind Institute, Ecole Polytechnique Fédérale \\ de Lausanne (EPFL), 1015 Lausanne, Switzerland, ${ }^{3}$ Department of Neuropathology, Graduate School of Medicine, University of Tokyo, Bunkyo-ku Tokyo \\ 113-0030, Japan, and ${ }^{4}$ Department of Neuroscience and ${ }^{5}$ Institute for Translational Neuroscience, University of Minnesota, Minneapolis, Minnesota 55102
}

Accumulation of misfolded $\alpha$-synuclein $(\alpha \mathrm{S})$ is mechanistically linked to neurodegeneration in Parkinson's disease (PD) and other $\alpha$-synucleinopathies. However, how $\alpha$ S causes neurodegeneration is unresolved. Because cellular accumulation of misfolded proteins can lead to endoplasmic reticulum stress/unfolded protein response (ERS/UPR), chronic ERS could contribute to neurodegeneration in $\alpha$-synucleinopathy. Using the A53T mutant human $\alpha$ S transgenic (A53T $\alpha S$ Tg) mouse model of $\alpha$-synucleinopathy, we show that disease onset in the $\alpha \mathrm{S}$ Tg model is coincident with induction of ER chaperones in neurons exhibiting $\alpha \mathrm{S}$ pathology. However, the neuronal ER chaperone induction was not accompanied by the activation of phospho-eIF $2 \alpha$, indicating that $\alpha$-synucleinopathy is associated with abnormal UPR that could promote cell death. Induction of ERS/UPR was associated with increased levels of ER/microsomal (ER/M) associated $\alpha \mathrm{S}$ monomers and aggregates. Significantly, human PD cases also exhibit higher relative levels of ER/M $\alpha \mathrm{S}$ than the control cases. Moreover, $\alpha \mathrm{S}$ interacts with ER chaperones and overexpression of $\alpha \mathrm{S}$ sensitizes neuronal cells to ERS-induced toxicity, suggesting that $\alpha \mathrm{S}$ may have direct impact on ER function. This view is supported by the presence of ERS-activated caspase-12 and the accumulation of ER-associated polyubiquitin. More important, treatment with Salubrinal, an anti-ERS compound, significantly attenuates disease manifestations in both the $A 53 T \boldsymbol{\alpha} S$ Tg mouse model and the adeno-associated virus-transduced rat model of $A 53 T \boldsymbol{\alpha} S$ dependent dopaminergic neurodegeneration. Our data indicate that the accumulation $\alpha \mathrm{S}$ within ER leads to chronic ER stress conditions that contribute to neurodegeneration in $\alpha$-synucleinopathies. Attenuating chronic ERS could be an effective therapy for PD and other $\alpha$-synucleinopathies.

\section{Introduction}

Parkinson's disease (PD) is the second most common neurodegenerative disease after Alzheimer's disease. While the etiology of $\mathrm{PD}$ is unknown in most cases, degenerating neuronal populations in PD exhibit $\alpha$-synuclein $(\alpha S)$ abnormalities and mutations in the $\alpha S$ gene cause familial PD, indicating that the $\alpha \mathrm{S}$ abnormalities are mechanistically linked to pathogenesis of PD and other $\alpha$-synucleinopathies (Vila and Przedborski, 2004; Obeso et al., 2010). While abnormal oligomerization/aggregation of $\alpha \mathrm{S}$ is most often implicated as a pathogenic event in $\alpha$-synucleinopathy, how $\alpha \mathrm{S}$ causes neurodegeneration in vivo is

Received Oct. 24, 2011; revised Dec. 31, 2011; accepted Jan. 12, 2012.

Author contributions: E.C., P.C., B.L.S., and M.K.L. designed research; E.C., P.C., Y.L., O.P., B.L.S., and M.K.L. performed research; 0.P., J.C.T., and T.I. contributed unpublished reagents/analytic tools; E.C., P.C., B.L.S., and M.K.L. analyzed data; E.C., P.C., B.L.S., and M.K.L. wrote the paper.

This work was supported by National Institutes of Health Grants NS038065, NS0380377, NS055776, and ES017384 (M.K.L.); and Swiss National Science Foundation, Grant 31003A_120653 (B.L.S.).We thank Drs. David Ron, Darren Moore, and Shaida Andrabi for helpful discussions. We also thank Dr. Virginia Lee for kindly providing the syn303 antibody.

The authors declare no competing financial interests.

This article is freely available online through the J Neurosci Open Choice option.

Correspondence should be addressed to Dr. Michael K. Lee, Department of Neuroscience, University of Minnesota, 21016 th Street SE., Minneapolis, MN 55102. E-mail: mklee@umn.edu.

E. Colla's present address: Laboratory of Neurobiology, Scuola Normale Superiore, I-56126 Pisa, Italy.

DOI:10.1523/JNEUROSCI.5367-11.2012

Copyright $\odot 2012$ the authors $\quad 0270-6474 / 12 / 323306-15 \$ 15.00 / 0$ poorly understood. Consequently, approaches to halt or prevent $\alpha$-synucleinopathy and related neurodegeneration are currently lacking.

Cellular accumulation of misfolded proteins can lead to chronic endoplasmic reticulum stress (ERS) and trigger an integrated cellular response called unfold protein response (UPR), which attempts to protect cells from accumulation of toxic misfolded proteins. However, chronic unabated ERS leads to the activation of cell death cascade (Ron and Walter, 2007). Potential involvement of chronic ERS in $\alpha \mathrm{S}$-dependent neurodegeneration was first demonstrated in a PC12 cell model of $\alpha$ S toxicity (Smith et al., 2005). A recent series of reports suggests that increased $\alpha \mathrm{S}$ expression can cause ER stress in yeast and other cells by interrupting Rab-dependent ER to Golgi membrane trafficking (Cooper et al., 2006). However, except for a limited number of neuropathological studies suggesting the activation of UPR in human PD cases (Hoozemans et al., 2007), it is unknown if $\alpha \mathrm{S}$ abnormalities can directly cause ER stress in vivo. More important, it is not known whether ERS is important for onset/progression of disease manifestation in vivo.

To determine whether ERS is involved in $\alpha S$-dependent neurodegeneration in vivo, we analyzed the activation of ERS pathways as a function of $\alpha$ S expression and $\alpha$-synucleinopathy in the transgenic (Tg) mouse model expressing various human $\alpha \mathrm{S}$ variants. We show that $\alpha$-synucleinopathy is coincident with induction 
of ERS, abnormal UPR signal, and activation of ERS-induced cell death pathway in vivo. Significantly, $\alpha$-synucleinopathy was also associated with increase in $\mathrm{ER} /$ microsomal (ER/M) $\alpha \mathrm{S}$ aggregates and polyubiquitin. More important, Salubrinal, an anti-ERS agent, attenuates disease manifestations in the $A 53 T \alpha S T g$ mouse model and in a rat adeno-associated virus $(A A V)$ model of $\alpha \mathrm{S}$ toxicity. We propose that increased ER accumulation of $\alpha \mathrm{S}$ and $\alpha \mathrm{S}$ aggregates triggers the chronic ERS that contributes to neurodegeneration. Further, agents that protect cells from chronic ERS could be developed as disease-modifying therapeutics for PD and other $\alpha$-synucleinopathies.

\section{Materials and Methods}

\section{Tg mouse models}

Tg mice expressing high levels of $W T$ or mutant (A53T and A30P) $\alpha S$ under the control of the mouse prion protein promoter have been described previously (Lee et al., 2002; Martin et al., 2006; Wang et al., 2008). Mice expressing A53T $\alpha$ S [line G2-3(A53T)] develop fatal neurological disease at $\sim 12$ months of age which rapidly progresses to end state within $14-21 \mathrm{~d}$ of onset. At disease onset, the mice exhibit neuronal $\alpha$-Syn and ubiquitinated aggregates/inclusions(Lee et al., 2002), degeneration of axons (Martin et al., 2006; Wang et al., 2008), and neuronal loss (Martin et al., 2006). For this study, early-stage-affected $A 53 T \alpha S \mathrm{Tg}$ mice exhibit slight instability, bradykinesia, and ataxia. The end-stage mice were defined by the onset of the paralysis. Presymptomatic mice were 10-14 months old and free from any motor dysfunction. Age-matched nTg littermates, $A 30 P \alpha S$ and $W T \alpha S$ Tg mice, were also used. SOD1 (G37R) Tg mice were provided by Dr. D.R. Borchelt (Department of Neuroscience, University of Florida).

For the Salubrinal treatment, a cohort of G2-3(A53T) Tg mice $(n=$ 27 ) was randomly assigned to either Vehicle $(n=12)$ or Salubrinal $(n=$ 14) group using GraphPad StatMate. At 12 months of age, six Tg mice developed neurological symptoms. Remaining asymptomatic G2$3(A 53 T) \mathrm{Tg}$ mice were administered $1.5 \mathrm{mg} / \mathrm{kg}$ of Salubrinal (Axxora) $(n=11)$ or vehicle $(n=10)$, three times per week via oral gavages for $\sim 6$ months by a lab staff blinded to the experimental conditions. Salubrinal was first dissolved in DMSO and then diluted 20 times with milk. Mice that became sick during the treatment were taken at end stage as described above. All animal study methods were approved in full by the Institutional Animal Care and Use Committee of the Johns Hopkins University and consistent with the requirements of the National Institutes of Health Office of Laboratory Animal Welfare Policy.

Tissues from human PD and control cases

Brain tissues were obtained from the Brain Resource Center (Department of Pathology, Johns Hopkins University School of Medicine, Baltimore, MD). The pathological characterizations of the tissues were done as described previously (Pletnikova et al., 2005). The diagnosis and postmortem delay times for the human tissues are listed in the Table 1.

\section{Cell lines}

Inducible BE(2)-M17 neuroblastoma cell line was created using the Tetresponsive system. Briefly, full-length cDNA for WT or A53T mutant $\alpha \mathrm{S}$ was cloned into $p c D N A 4 / T O$ tetracycline-regulated expression vector (Invitrogen). Constructs including control plasmid $p c D N A 4 / T O / l a c Z$ were cotransfected into $B E(2)-M 17$ Tet-on cells with $p c D N A 6 / T R$ (Invitrogen) and selected using $10 \mu \mathrm{g} / \mathrm{ml}$ blasticidin and $200 \mu \mathrm{g} / \mathrm{ml}$ Zeocin. Clones of cells were induced to express $\alpha \mathrm{S}$ or LacZ by addition of $1 \mu \mathrm{m}$ doxycycline. For the toxicity studies, M17 cells were induced to express the transgene ( $\alpha \mathrm{S}$ or LacZ) by treating with doxycycline for $3 \mathrm{~d}$, followed by increasing concentrations of tunicamycin and thapsigargin (SigmaAldrich). Cell toxicity was assayed using the Cell Proliferation Kit II (XTT) (Roche). SH-SY5Y cell lines expressing mouse $\alpha$ S or $\beta$ S were also used (Li et al., 2004b; Li and Lee, 2005).

\section{Microsomes preparation and membrane floatation}

For subcellular fractionation of ER membrane-enriched microsomes (Cox and Emili, 2006), fresh tissues were homogenized in a 1:10 (w/v) volume of lysis buffer with the following (in mM): 250 sucrose, 20 HEPES,
Table 1. Diagnosis, age, and postmortem delay of human brain tissues used

\begin{tabular}{lllc}
\hline & Diagnosis & Age (years) & PMD (h) \\
\hline Controls without BrSt LBDs & Control & 66 & 10 \\
& Control & 74 & 4 \\
& AD & 78 & 4 \\
& AD & 88 & 14 \\
& AD & 80 & 9 \\
& AD & 92 & 7 \\
& AD & 78 & 8 \\
PD with BrSt LBDs & AD & 69 & 10.5 \\
& PD w/dementia & 75 & 9 \\
& PD w/dementia & 83 & 5 \\
& PD w/dementia/AD & 80 & 16 \\
& PD/AD probable & 82 & 5 \\
& PD/AD possible & 75 & 9 \\
& PD/AD possible & 85 & 14 \\
& PD/diffuse A $\beta$ deposits & 77 & 16 \\
& PD/LBD & 90 & 16 \\
& PD/AD/LBD & 84 & 12 \\
& PD/AD & 64 & 21 \\
& PD & 85 & 14 \\
PD & 76 & 18 \\
\hline
\end{tabular}

All PD cases exhibit brainstem (BrSt) $\alpha$-synuclein pathology level of at least 10 where as the controls are 2 or less (Pletnikova et al., 2005). AD, Alzheimer's disease; LBD, Lewy body dementia; MID, mutiple infarct dementia; PD, Parkinson's disease; PMD, postmortem delay.

$10 \mathrm{KCl}, 1.5 \mathrm{MgCl}_{2}, 2 \mathrm{EDTA}$, protease-inhibitor mixture) using a Teflon pestle homogenizer. Initial homogenates were centrifuged at $1000 \times g$ to remove nuclei and unbroken cells. The resulting supernatant was centrifuged at $10,000 \times g$ to remove mitochondria and the postmitochondrial supernatant was centrifuged at $100,000 \times g$. The pellet was used as microsome fraction while the supernatant was used as pure cytosol. The microsome pellets were washed once with lysis buffer and resuspended in $100 \mu \mathrm{l}$ of lysis buffer. To further enrich for the ER content, the microsome preparation were applied to a $0.2 \mathrm{~m} / 0.8 \mathrm{~m} / 2 \mathrm{M}(\mathrm{v} / \mathrm{v}$ ratio $3: 4: 4)$ discontinuous sucrose gradient (Croze and Morré, 1984) and centrifuged at $90,000 \times g$ for $2 \mathrm{~h}$ in a swinging bucket rotor (Sorvall TH-641). The interface between 0.8 and $2 \mathrm{M}$ was collected, diluted with sucrose-free lysis buffer, and centrifuged at $110,000 \times g$ for $45 \mathrm{~min}$, and the final pellet was collected (ER-enriched microsome).

Pure mitochondria were obtained by discontinuous gradient fractionation of the crude mitochondria pellet (P10) (Vijayvergiya et al., 2005). The pellet was resuspended in lysis buffer and then layered on top of a chilled 7.5/10\% (w/v) discontinuous Ficoll gradient. The samples were centrifuged at $24,000 \mathrm{rpm}$ for $24 \mathrm{~min}$ at $4^{\circ} \mathrm{C}$ in a swinging bucket rotor (Sorvall TH-641). The purified mitochondrial pellet was resuspended in $200 \mu \mathrm{l}$ of lysis buffer.

Pure nuclei were isolated starting from the crude nuclei pellet (P1) (Cox and Emili, 2006) using a sucrose gradient. Briefly, crude nuclei pellet were washed once and then resuspended in a $2 \mathrm{~m}$ sucrose solution made in sucrose-free lysis buffer. This pellet was then layered at the top of a $2 \mathrm{M}$ sucrose gradient and spun in a swinging bucket ultracentrifuge at $80,000 \times g$ for $35 \mathrm{~min}$. After aspirating the supernatant, the pellet that includes pure nuclei was resuspended in the original lysis buffer.

For fractionation by membrane floatation (Ding et al., 2002), microsomes (P100) were resuspended in $0.42 \mathrm{ml}$ of $60 \%$ iodixanol solution (Sigma-Aldrich) and overlayered with a discontinuous gradient containing $2.5 \mathrm{ml}$ of $25 \%$ and $0.1 \mathrm{ml}$ of $5 \%$ iodixanol. Samples were centrifuged at $200,000 \times g$ for $2 \mathrm{~h}$ in a swinging bucket rotor (Sorvall TH-641) and the fractions were collected from the $5 / 25 \%$ (membrane-bound) and $25 / 60 \%$ (membrane-free) interfaces and analyzed.

\section{Proteinase K protection assay}

The microsome fractions were treated with or without $50 \mu \mathrm{g} / \mathrm{ml}$ proteinase K (PK; Roche) and 1\% Triton X-100 for 20 min on ice. The reaction was stopped by the addition of $2 \mathrm{~mm}$ (final concentration) phenylmethylsulfonyl fluoride. 
Immunoblotting and dot-blot analysis

Immunoblot and dot-blot analysis of mice and human brain tissues were performed as previously described (Lee et al., 2002; Li et al., 2004b; Li et al., 2005; Martin et al., 2006; Wang et al., 2008). For semiquantitative analysis of protein expression, the chemiluminescence signal associated with antibody binding (Pierce) was captured using the Bio-Rad Molecular Imager ChemiDoc XRS + System or on X-ray films. The intensities of the immunoreactive bands were determined using the Quantity One software (Bio-Rad). For dot-blot analysis, lysates were spotted directly on the nitrocellulose membrane and allowed to dry completely. Immunoreactivity was visualized using chemiluminescence detection (Pierce) after incubations with the appropriate horseradish peroxidase-conjugated secondary antibody, using a CCD-based Bio-Rad Molecular Imager ChemiDoc XRS + System or x-ray films. The intensities of the immunoreactive bands were determined by densitometry and the Quantity One software (Bio-Rad).

The following antibodies were used: grp78, grp94, proline disulfide isomerase (PDI), calnexin, syntaxin 6 (Stressgen); p-eIF2 $\alpha$, eIF2 $\alpha$, caspase-9, caspase-3, synaptotagmin (Cell Signaling Technology); syn-1, cytochrome $c$ (BD Transduction Laboratories); ATF4, CHOP, p38, caspase-12, ubiquitin, cathepsin D (Santa Cruz Biotechnology); VDAC, NeuN, (Abcam); pser129- $\alpha$ S (Fujiwara et al., 2002); syn303 (Duda et al., $2000)$; and $\beta S$ (Oncogene).

\section{Immunoprecipitation}

Immunoprecipitation was conducted using Seize X Protein G Immunoprecipitation Kit (Pierce) as previously described (Li et al., 2004a,b). Briefly, $1 \mu$ g of syn- 1 or grp78 antibody was cross-linked using DSP 2 mM to protein $\mathrm{G}$ agarose and used for immunoprecipitation. Bound proteins were freed from the beads by SDS-sample buffer before fractionation by SDS-PAGE.

\section{Immunohistochemistry and confocal microscopy}

For immunohistochemical analysis, mice were fixed with $4 \%$ paraformaldehyde (PFA), serially frozen-sectioned $(30 \mu \mathrm{m})$, and immunostained for $\mathrm{DAB}$ detection and for double immunofluorescence as previously described (Liu et al., 2008).

For the quantitative analysis of ER chaperones' expression in neurons accumulating $\alpha \mathrm{S}$ abnormalities, fluorescence quantification of ER stress chaperones' signal was done using ImageJ software (National Institutes of Health). Mean values correspond to signal intensity of grp78/BIP or grp94 after subtraction of the nuclei fluorescence and normalized with the respective neuronal area. Values are expressed as the percentage of intensity of neurons in the same section that are syn303 or pS129- $\alpha \mathrm{S}$ negative.

\section{Immunoelectronic microscopy}

Diseased A53T $\alpha S$ mice and nTg littermates were perfused with 4\% PFA/ $0.1 \%$ glutaraldehyde. Brain and spinal cord $(\mathrm{SpC})$ sections $(50 \mu \mathrm{m})$ were stained with pS129- $\alpha$ S antibody as described above, labeled with $6 \mathrm{~nm}$ gold particle-conjugated secondary antibody, and embedded for electron microscopy. Samples were visualized using a Hitachi 7600 transmission electron microscope.

\section{AAV2/6 plasmid construction, production, and titration}

Human $\alpha S$ gene (GenBank accession no. NM_000345) carrying the A53T mutation was inserted in a $p A A V-p g k-M C S-W P R E$ backbone modified from a $p A A V-c m v-M C S$ (Stratagene), using standard cloning procedures. The noncoding $p A A V$-pgk-MCS-WPRE backbone was used to produce an empty control vector.

Recombinant pseudotyped $A A V 2 / 6$ vectors were produced, purified, and titrated as described previously (Dusonchet et al., 2009). Briefly, we measured the integration of transcriptionally active transgene copies at $48 \mathrm{~h}$ in HEK293T cells and obtained the following titers: AAV2/6-pgk$\alpha S y n-A 53 T-W P R E=6.4 \times 10^{9} \mathrm{TU} / \mathrm{ml}, A A V 2 / 6-p g k-M C S-W P R E=$ $1.5 \times 10^{10}$ transducing units $(\mathrm{TU}) / \mathrm{ml}$.

\section{$\mathrm{AAV}$-rat model of $\mathrm{A} 53 \mathrm{~T} \alpha \mathrm{S}-$ dependent dopaminergic} neurodegeneration

Female adult Sprague Dawley rats (Charles River Laboratories), weighing $\sim 200 \mathrm{~g}$, were used in accordance with Swiss legislation and the European
Community Council directive (86/609/EEC) for the care and use of laboratory animals. For stereotaxic injections, the animals were deeply anesthetized with a mixture of xylazine/ketamine and placed in a stereotaxic frame (David Kopf Instruments). Two microliters of viral preparation were injected in the right brain hemisphere using a $10 \mu \mathrm{l}$ Hamilton syringe with a 34 gauge blunt tip needle connected to an automatic pump (CMA Microdialysis) at a speed of $0.2 \mu \mathrm{l} / \mathrm{min}$. The needle was left in place for 7 additional minutes before being slowly withdrawn. The substantia nigra pars compacta $(\mathrm{SNpc})$ was targeted at the following coordinates: anteroposterior, $5.2 \mathrm{~mm}$; mediolateral, $2.0 \mathrm{~mm}$ relative to bregma; and dorsoventral, $7.8 \mathrm{~mm}$ relative to skull surface. For both the AAV2/6-pgk$\alpha S$-A53T-WPRE and AAV2/6-pgk-MCS-WPRE vectors, the total injected vector dose was $1.0 \times 10^{7}$ TUs.

Rats were treated with $1.5 \mathrm{mg} / \mathrm{kg}$ of Salubrinal (Alexis Biochemical) three times per week. The compound was solubilized in 5\% DMSO mixed with chocolate milk. The rats were voluntarily drinking the Salubrinal solution from the tip of a $1 \mathrm{ml}$ tuberculin syringe (Braun). Control animals were similarly treated with vehicle containing only DMSO.

\section{Behavioral analysis of AAV-rat model}

Cylinder test. Spontaneous forelimb use during explorative behavior was assessed in the cylinder test (Schallert et al., 2000). Animals were placed in a transparent cylinder with a $20 \mathrm{~cm}$ diameter and movements were recorded using a video camera placed above the cylinder (5-15 min, depending on the level of animal activity). Paw touches on the cylinder wall were scored for each forelimb. Data are expressed as the percentage of left forelimb touches versus the total number of paw touches.

Drug-induced rotametry. Apomorphine-induced rotational behavior was monitored for $40 \mathrm{~min}$ using automated rotameter bowls (Rotoscan, Rotometer v5.06; Omnitech) following subcutaneous injection of apomorphine- $\mathrm{HCl}(0.1 \mathrm{mg} / \mathrm{kg})$ (Sigma). Animal rotational activity is expressed as the net number of counterclockwise turns per minute.

Quantitative neuropathological analysis of AAV-rat model. Rats were killed and tissue processed for quantitative neuropathological analysis as described previously (Dusonchet et al., 2009).

\section{Quantification of neurons displaying Golgi fragmentation}

Coronal $40-\mu \mathrm{m}$-thick sections of the midbrain, immunostained for $\mathrm{TH}$, $\alpha \mathrm{S}$, and GM130, were analyzed using a laser scanning confocal microscope (Zeiss LSM700). Golgi morphology was evaluated in the entire population of nigral TH-positive neurons expressing $\alpha \mathrm{S}$, across eight sections per animal, in a blind fashion (three animals per group). The Golgi apparatus was considered as "fragmented" when a completely scattered and punctuated GM130 signal was observed. When tubular structures were still present, the Golgi was considered to be in an intermediate stage of fragmentation and the cell was not attributed to any of the "normal" or "fragmented" populations.

\section{Statistical analysis}

All values are expressed as the mean \pm SEM. Differences between means were analyzed by Student's $t$ test and one- or two-way ANOVA with the Bonferroni's post test (Prism; Graph Pad Software). For survival curve log-rank (Mantel-Cox) test was used (Prism; Graph Pad Software).

For the rat AAV model, data are expressed as mean \pm SEM. Statistical analysis of behavioral data were performed using one-way ANOVA (Statistica; StatSoft) followed by a Newman-Keuls post hoc test. Statistical analysis of striatal dopaminergic (DAergic) innervation and stereological quantification of the nigral TH-positive neurons was performed using a one-way ANOVA (Statistica; StatSoft) followed by an LSD Fisher post hoc test. Statistical analysis of Golgi fragmentation was performed using oneway ANOVA followed by a Tukey's post hoc test (Statistica; StatSoft).

\section{Results}

Increase in ER chaperones with disease in A53T $\alpha S \mathrm{Tg}$ mice is not accompanied by activation of phospho-eIF $2 \alpha$

Cell culture studies implicate ERS in $\alpha \mathrm{S}$ cell toxicity (Smith et al., 2005; Cooper et al., 2006). While PD is associated with signs of UPR (Hoozemans et al., 2007), a variety of antemortem and post- 
A

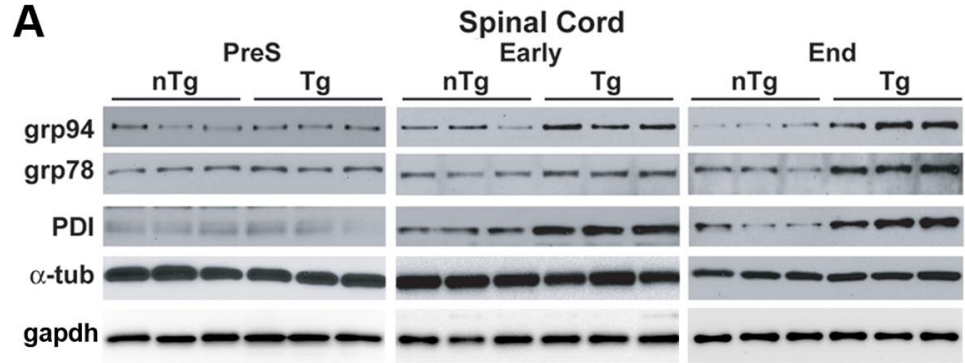

B

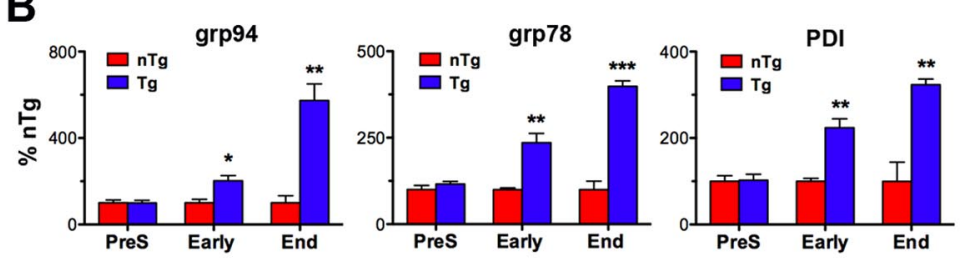

C
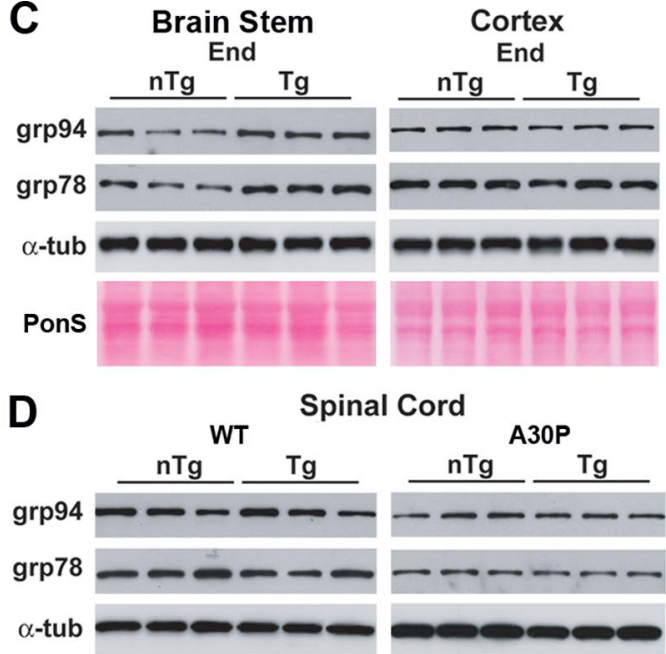
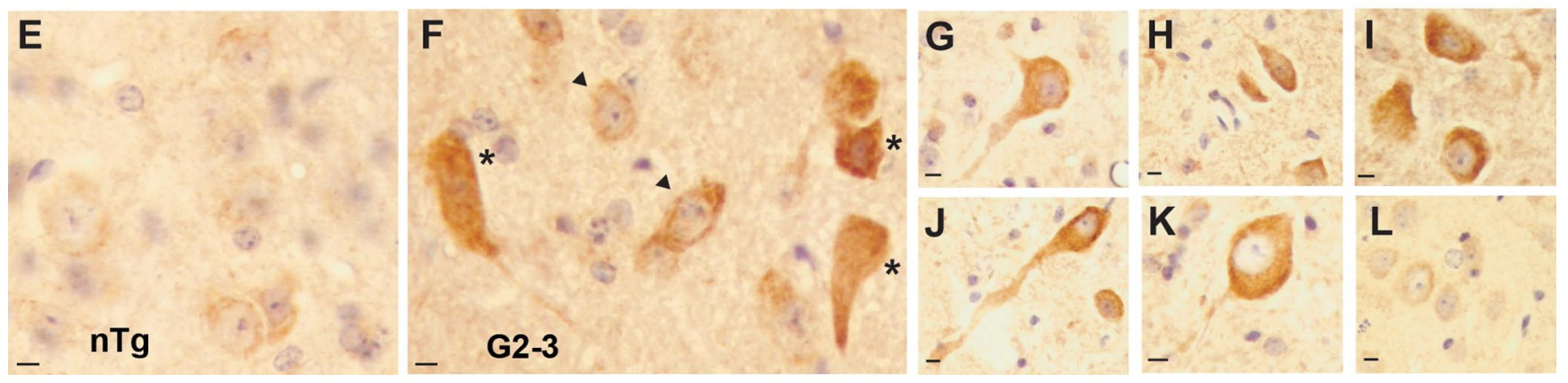

Figure 1. Accumulation of ER chaperones with the onset of disease in $A 53 T \alpha S \operatorname{Tg}$ mice. $A, S p C$ lysates from $A 53 T \alpha S \operatorname{Tg}$ mice at different disease stages and littermate $\mathrm{nTg}$ mice were immunoblotted for grp78, grp94, and PDI. The levels of ER chaperones in symptomatic Tg mice (Early and End stages) are clearly higher compared with the levels in the presymptomatic (PreS) or nTg animals. $B$, Quantitative analysis of various chaperone levels in $\mathrm{Tg}$ and $\mathrm{nTg}$ mice. The chaperone levels were normalized to the mean of $\mathrm{nTg}$ mice. All values represent mean \pm SEM with $n=6$ per group. ${ }^{* * *} p<0.001,{ }^{* *} p<0.01,{ }^{*} p<0.05$ compared with the littermate $n T$ Tg mice, Student's t test. C, Lysates from Brainstem, another area that develops pathology, and Cortex, a pathology-free region, from end-stage $\mathrm{Tg}$ and $\mathrm{nTg}$ mice were analyzed for grp78 and grp94. There is significant increase in the ER chaperones in the Brainstem (grp94, 174.07 $\pm 4.08 \%$ of $\mathrm{nTg}, p<0.01 ; \mathrm{grp} 78$, $165.31 \pm 5.54$ of $\mathrm{nTg}, p<0.05 ; n=6$ per group) but not in Cortex. D. Levels of ER chaperones in the Spinal Cord of Tg mice (WT and A30P $\alpha$ S, 14-16 months old) that overexpress $\alpha S$ without pathology are not different from age-matched nTg mice. Also shown are $\alpha$-tubulin (DM1A, $\alpha$-tub) and Ponceau $S$ staining (Pons) for controls. $E$-L, Immunohistochemical analysis of grp78 expression in $\mathrm{nTg}(\boldsymbol{E})$ and end-stage $A 53 T \alpha \mathrm{STg}$ mice $(\boldsymbol{F}-\boldsymbol{L})$. Areas are SpC $(\boldsymbol{E}-\boldsymbol{G}), \mathrm{Cbl}(\boldsymbol{H}, \boldsymbol{I}), \mathrm{BrSt}(\boldsymbol{J}, \boldsymbol{K})$, and $\mathrm{Ctx}(\boldsymbol{L})$. Neurons exhibiting intense grp78 expression (denoted by asterisks) show abnormal morphology. Surrounding normal neurons show low basal levels of grp78 staining (arrowheads). Sections were counterstained with H\&E. Scale bars, $10 \mu \mathrm{m}$.

mortem factors can activate UPR. Thus, it is not known if there are direct links between $\alpha$ S-dependent neurodegeneration and ERS in vivo. To address this issue, we analyzed an $A 53 \mathrm{~T} \alpha \mathrm{S} \mathrm{Tg}$ mouse model of $\alpha$-synucleinopathy (Lee et al., 2002) for the presence of ERS/UPR activation.

First, we examined if $A 53 \mathrm{~T} \alpha S \mathrm{Tg}$ mouse model exhibited increase in the expression of ER chaperones as grp94, grp78, and PDI. These markers are widely used indicators of ERS/UPR activation (Ron and Walter, 2007). Quantitative immunoblot analysis of pathologically affected regions [ $\mathrm{SpC}$ and brainstem $(\mathrm{BrSt})$ ] show increased levels of grp78, grp94, and PDI with the progression of $\alpha$-synucleinopathy (Fig. 1A,B). In SpC, increases in the ER chaperone levels were coincident with the onset of neurological abnormalities in the early symptomatic mice, which are characterized by a moderate wobbling gate (Martin et al., 2006). In addition, parallel analysis of BrSt from an end-stage $A 53 \mathrm{~T} \alpha \mathrm{S} \mathrm{Tg}$ mouse (Fig. 1C) shows significant increase in both grp94 (174 \pm $4.1 \%$ of nTg, $p<0.01)$ and grp78 ( $165.3 \pm 5.5 \%$ of nTg, $p<0.05)$ levels. The levels of ER chaperones in the cortex (Fig. 1C), a region with high levels of mutant $\alpha \mathrm{S}$ expression without severe neuropathology, were comparable between the groups of mice. Consistent with the increased expression of ER chaperones, spinal cords of clinically affected mice show activation of the X-box- binding protein $1(x b p 1)$, a transcription factor involved in transcriptional induction of the ER chaperones at the early stage of disease process (Fig. 2A).

To further establish that induction of ER chaperones and UPR activation occurs with the presence of $\alpha$ S-associated neuropathology rather than simple interaction between aging and/or nonpathologic $\alpha$ S overexpression, we examined the expression of the ER chaperones in the $\alpha \mathrm{S}$ overexpressing Tg mouse lines that do not develop neuropathology [line $\mathrm{O}_{2}(\mathrm{~A} 30 \mathrm{P})$ and line I22 (WT) (Lee et al., 2002)]. The ER chaperone levels in SpC of aged (14 months) A30P mice and WT $\alpha S$ Tg mice were not different from the nTg littermates (Fig. $1 D$ ). Combined with the fact that the ER chaperone levels in the cortex of end-stage $A 53 \mathrm{~T} \alpha \mathrm{S} \mathrm{Tg}$ mice, these results show that the onset of $\alpha$-synucleinopathy and neurological abnormalities are intimately linked to the presence of ERS in brain. While the studies of using simpler systems (Cooper et al., 2006; Gitler et al., 2008) predicted that high levels of $\alpha S$ expression alone would be sufficient to cause ERS response, in mammalian brain, overt $\alpha$-synucleinopathy and/or neurodegeneration appears to be a prerequisite for the induction of ERS.

In addition to the transcriptional induction of ER chaperones, UPR also involves general inhibition of protein translation during ER stress to reduce demand on the cell-folding machinery 


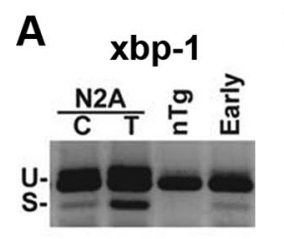

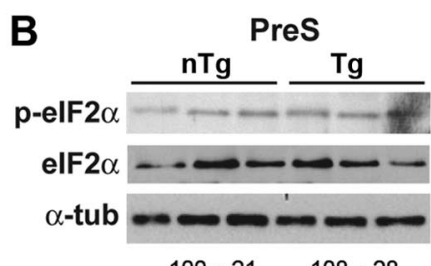

$100 \pm 21$

$108 \pm 28$

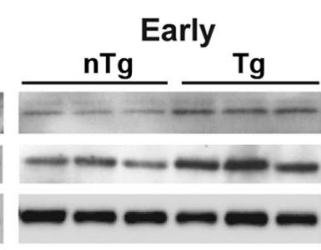

$100 \pm 36$

$102 \pm 18$

p-elF $2 \alpha /$ elF $2 \alpha$ ratio ( $\% \mathrm{nTg}$ )

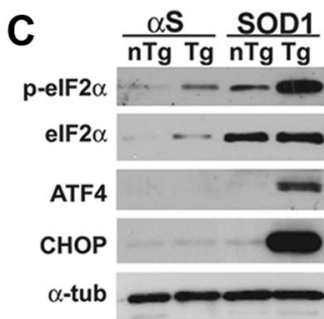

Figure 2. Alternative splicing of XBP-1 mRNA with the lack of phospho-elF2 $\alpha$ activation with $\alpha$-synucleinopathy in the $A 53 T \alpha S$ Tg mice. $A$, Splicing of $x b p 1$ mRNA mediated by the inositolrequiring enzyme, IRE1, leads to $x b p 1 \mathrm{mRNA}$ variant encoding the active protein. RT-PCR analysis for $x b p 1 \mathrm{mRNA}$ shows the presence of alternatively spliced variant ( $S$ ) in the $S p C$ from early-stage G2-3(A53T) mice but only the unspliced variant (U) is present in SpC from nTg mice. Also shown are control ( $($ ) and tunicamycin-treated (T) N2A cells. $\boldsymbol{B}$, Western blot analysis for phospho-elF2 $\alpha$ (p-elF2 $\alpha$ ) and total elF2 $\alpha$ (elF2 $\alpha$ ) expression in SpC from mice at various disease stages. Also shown are the $\alpha$-tub (DM1A) blots used for controls. The numbers below show the ratios of p-elF2 $\alpha$ /total elF $2 \alpha$, as a percentage of nTg levels. Values are mean \pm SEM $(n=6)$. C, Western blot analysis for phospho-elF2 $\alpha$ (p-elF2 $\alpha$ ), total elF2 $\alpha$ (elF2 $\alpha$ ), ATF4, and CHOP in $A 53 T \alpha S$ Tg and G37R-SOD1 Tg mice. Both mice were at end stage (paralyzed).

where the phosphorylation of the translation initiation factor, eIF $2 \alpha$, is thought to arrest general protein translation (Ron and Walter, 2007). Studies indicate that in cultured cells, phosphorylation of eIF $2 \alpha$ is important for maintaining cell viability during chronic ER stress conditions (Boyce et al., 2005). Analysis of the $A 53 T \alpha S$ Tg mice for the phosphorylated eIF $2 \alpha$ (p-eIF $2 \alpha$ ) show that $\alpha$-synucleinopathy was associated with increased levels of phospho-eIF $2 \alpha$. However, because the total eIF $2 \alpha$ was also elevated with $\alpha$-synucleinopathy, the relative levels of p-eIF $2 \alpha$ do not change with the progression of $\alpha$-synucleinopathy (Fig. $2 B$ ). Consistent with the lack of increased p-eIF2 $\alpha$ levels, analysis for CHOP and ATF4, which are selectively translated by p-eIF $2 \alpha$ (Ron and Walter, 2007), show that these components are not induced with the onset of $\alpha$-synucleinopathy (Fig. 2C). Absence of p-eIF $2 \alpha$ activation is not due to limitations in documenting the activation of this pathway in an in vivo model system since we are able to clearly show the increases in the levels of p-eIF $2 \alpha$, ATF4, and CHOP in the end-stage G37R mutant superoxide dismutase-1 (SOD1) Tg mice (Fig. 2C), where the disease is associated with activation of UPR (Saxena et al., 2009). Since the phosphorylation of eIF $2 \alpha$ is thought to protect cells from cell death induced by ERS (Boyce et al., 2005), our results indicate that the conditions in the $A 53 T \alpha S$ Tg mice are favorable for the activation of ERS-induced cell death cascade.

\section{ER chaperones are induced in neurons with $\alpha S$ pathology in A53T mice}

Upon establishing the specificity of ER stress with disease, we examined the cellular localization of the grp78 expression in brains of $A 53 T \alpha S \mathrm{Tg}$ mice in relation to $\alpha$-synucleinopathy. In nTg mice (Fig. $1 E$ ) and in cortical neurons (Fig. $1 L$ ), neuronal grp78 staining is sparsely distributed with punctuate perinuclear staining. In the end-stage $A 53 T \alpha S T$ g mice, a subset of neurons in the areas affected by $\alpha$-synucleinopathy, including deep cerebellar nuclei (Cbl), BrSt, and SpC, were highly reactive for grp78 (Fig. $1 \mathrm{~F}-\mathrm{K}$ ). Moreover, the neurons with increased grp78 expression showed abnormal morphology (such as hypertrophy, atrophy, and/or dystrophic neurites) while the neighboring neurons with a normal morphology exhibited lower levels of grp78 immunoreactivity (Fig. $1 F$ ). These results indicate that UPR occurs in neurons that are pathologically affected.

To further link $\alpha$-synucleinopathy with the presence of ERS, we asked whether the ER chaperone induction in the A53T $\alpha S \mathrm{Tg}$ mice occurs in neurons with $\alpha \mathrm{S}$ pathology. The $\alpha \mathrm{S}$ abnormalities were documented using either syn303 or the anti-pS129- $\alpha \mathrm{S}$ antibody and the ER chaperone expression was documented using antibodies to grp78 or grp94 (Ron and Walter, 2007). Confocal double-immunofluorescence microscopy reveals neurons in the affected areas and $A 53 T \alpha S$ Tg mice exhibiting the abnormal perikaryal and neuritic reactivity to syn303 or anti-pS129- $\alpha$ S (Fig. 3 ). While all neurons expressed ER chaperones as expected, neurons with abnormal $\alpha \mathrm{S}$ generally exhibited stronger grp78/94 immunoreactivity (Fig. $3 A, B, D$, asterisks). To confirm our qualitative observations, the intensity of grp78- or grp94-associated immunofluorescence was quantified in cells with and without abnormal $\alpha \mathrm{S}$ immunoreactivity. The results show that compared with ER chaperone levels in normal neurons within the same sections, neurons with abnormal $\alpha \mathrm{S}$ (either syn303+ or pS129$\alpha S+$ ) exhibited higher levels of ER chaperones (Fig. 3F).

Finally, immunoelectron microscopic analysis of diseased $A 53 T \alpha S$ Tg mice shows that a subset of $\mathrm{pS} 129-\alpha \mathrm{S}$ reactivity localizes on the ER membranes (Fig. 3G,I). More important, the ER morphologies in these neurons were highly abnormal with severely dilated ER cisternae, an ultrastructural indication of ER dysfunction in the $A 53 T \alpha S \mathrm{Tg}$ mice (Fig. 3G-J). Collectively, these results show that the ERS/UPR activation in $A 53 T \alpha S \mathrm{Tg}$ mice is selective for neurons exhibiting $\alpha \mathrm{S}$ pathology and the ER membranes show abnormal morphology in these neurons.

\section{$\alpha S$ and $\alpha S$ aggregate are associated with ER/M fraction}

Studies indicate that $\alpha \mathrm{S}$ can functionally impact multiple organelles (Gosavi et al., 2002; Smith et al., 2005; Cooper et al., 2006; Martinez-Vicente et al., 2008; Winslow et al., 2010). Given the colocalization of $\alpha$-synucleinopathy with ER chaperone activation and abnormal ER morphology (Fig. 3), ERS could be caused by direct effects of $\alpha \mathrm{S}$ or $\alpha \mathrm{S}$ aggregates on ER. As an initial test of this hypothesis, we examined whether $\alpha \mathrm{S}$ can biochemically cofractionate with the microsomes. We found that $\alpha \mathrm{S}$ and $\alpha \mathrm{S}$ aggregates indeed copurify with the microsomes (Fig. 4). Significantly, microsomal $\alpha \mathrm{S}$ was found in both Tg and $\mathrm{nTg}$ mice (Fig. $4 B, C$ ), as well as in human brain (Fig. $4 C$ ), indicating that $\alpha \mathrm{S}$ associates with ER under normal conditions. Association of $\alpha \mathrm{S}$ with ER is highly selective and is not related to the simple membrane-binding properties of synucleins since $\beta$ S, which also interacts with membranes (Narayanan and Scarlata, 2001), is not associated with the ER/M fractions (Fig. $4 B, C$ ). Lack of $\beta$ S in the $\mathrm{ER} / \mathrm{M}$ fractions from Tg mice is not because of competition by high levels of $\alpha \mathrm{S}$, since $\beta S$ does not associate with the microsomes in nTg mice and when overexpressed in SH-SY5Y cells (Fig. 4C). We also performed PK protection assay to determine whether microsomal $\alpha \mathrm{S}$ is bound to the membrane surface or translocates into the microsomes. Our studies show that the bulk of microsomal $\alpha \mathrm{S}$ are resistant to $\mathrm{PK}$ (Fig. 4D). This indicates that $\alpha \mathrm{S}$ is 

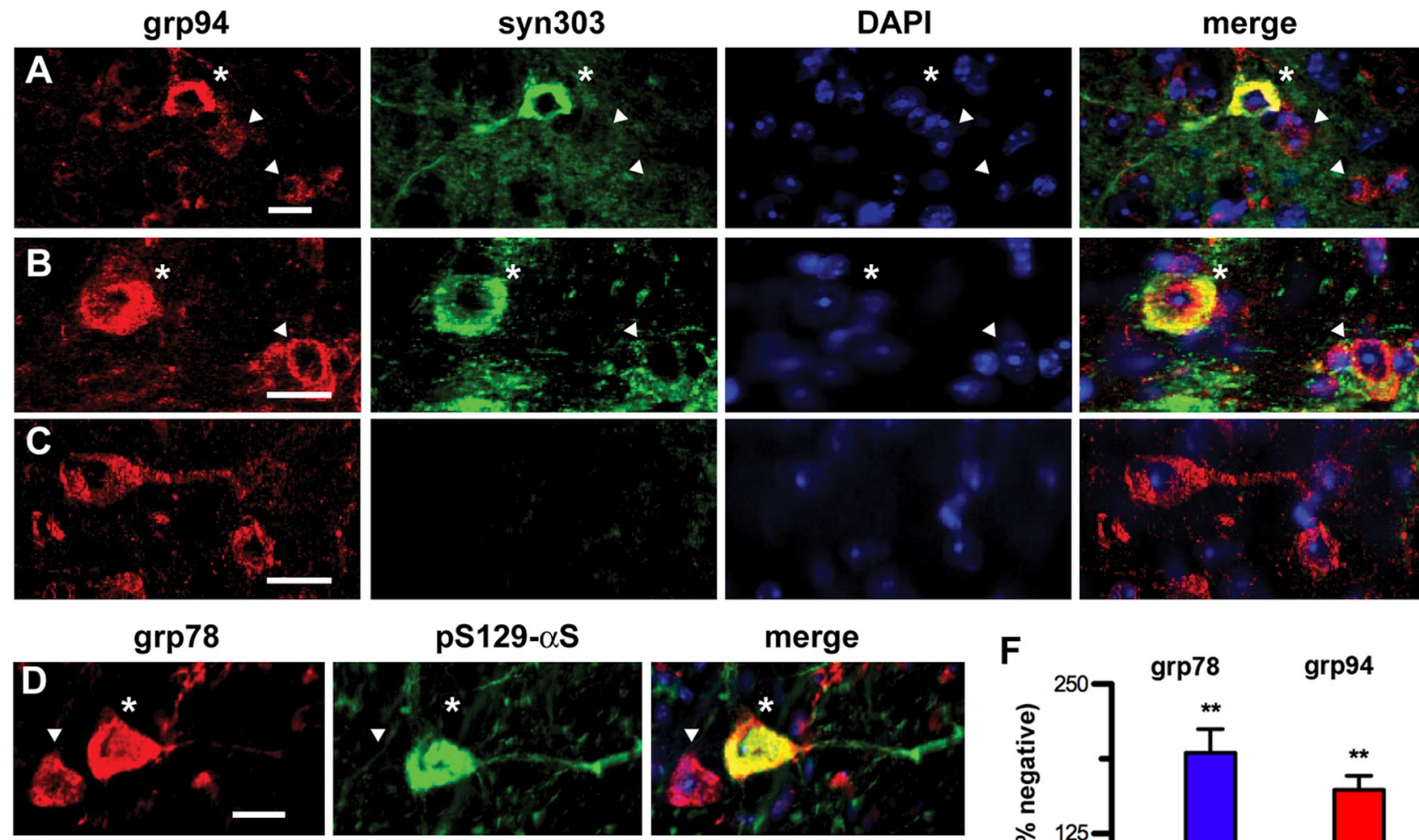

$\mathbf{F}$
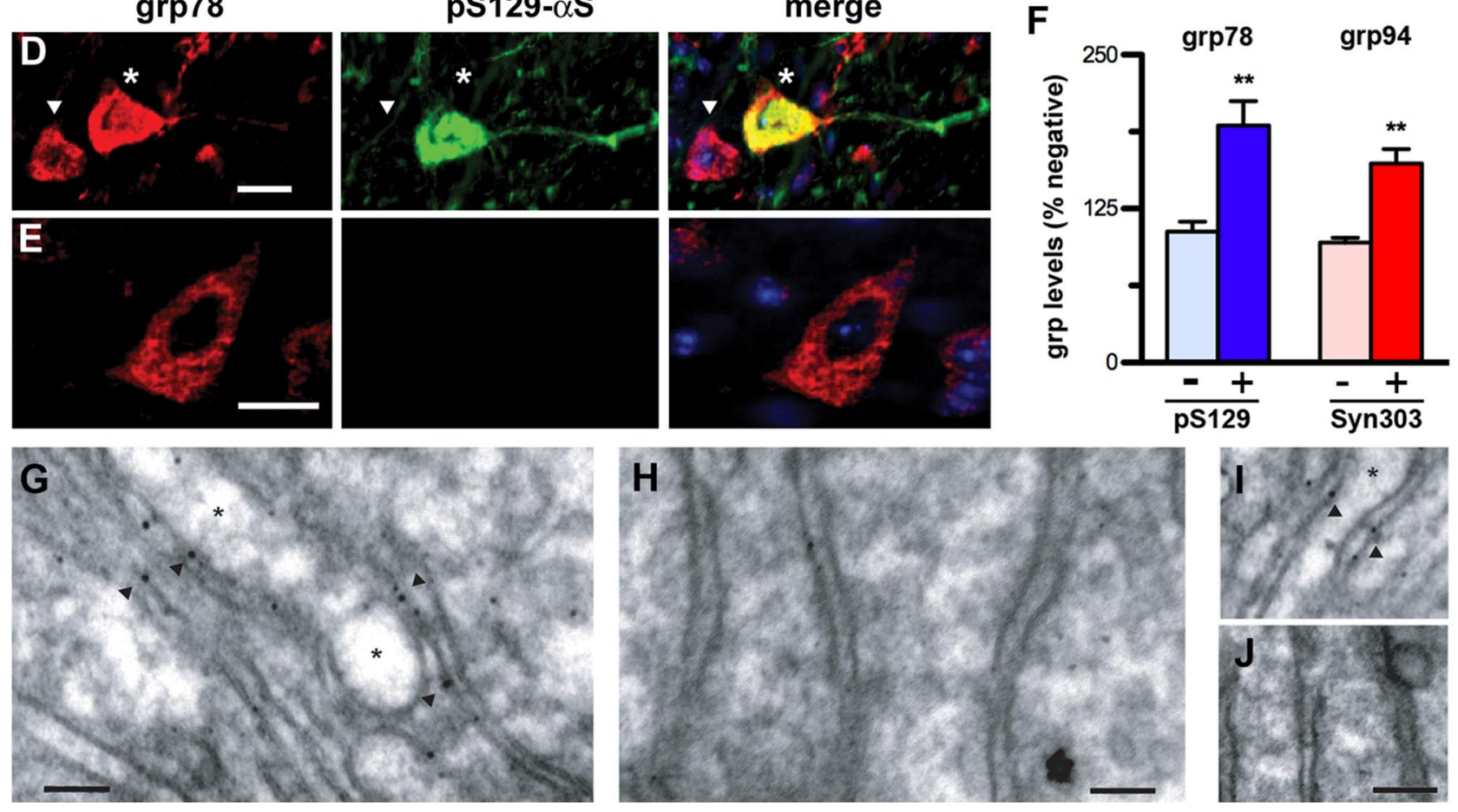

Figure 3. Induction of ER chaperones occurs in neurons with $\alpha$ S pathology. $\boldsymbol{A}-\boldsymbol{E}$, Confocal immunofluorescence colocalization of grp78 or grp94 with abnormal $\alpha S$. SpC $(\boldsymbol{A}, \boldsymbol{D}), \mathrm{BrSt}(\boldsymbol{B}, \boldsymbol{C})$, and Ctx $(\boldsymbol{E})$ sections from end-stage $A 53 T \alpha S \operatorname{Tg}$ mice $(\boldsymbol{A}, \boldsymbol{B}, \boldsymbol{D}, \boldsymbol{E})$ and $\mathrm{nTg}(\boldsymbol{C})$ mice were double-immunostained for grp94/syn303 (A-C) or for grp78/grp94 (KDEL)/pS129- $\alpha S$ (D, E). Nuclei were stained with DAPI. Neurons showing abnormal accumulation of syn303 or anti-pS129- $\alpha$ S are also associated with increased immunoreactivity for grp78/grp94 (asterisks). Neighboring neurons lacking abnormal $\alpha$ show lower basal grp78/grp94 immunoreactivity (arrowheads). $\boldsymbol{F}$, Quantitative analysis obtained using ImageJ software (National Institutes of Health) of neurons with $\alpha S$ abnormalities (stained for syn303 or pS129- $\alpha$ S) in BrSt and SpC of end-stage A53T $\alpha$ S Tg mice shows increased ER stress chaperones (grp78 for pS129- $\alpha$ S, grp94 for syn303). Values are mean \pm SEM; $n=$ 13 each for pS129- $\alpha$ S- and syn303-positive neurons and $n=25-31$ for negative neurons. ${ }^{* *} p<0.001$, Student's $t$ test. $\mathbf{G}-\boldsymbol{H}$, Immunogold labeling of $\alpha S$ aggregates shows $\alpha S$ aggregate association with the ER membrane in the diseased $A 53 T \alpha S$ mice. SpC sections from end-stage $A 53 T(G, I)$ and $n T g(H, J)$ were immunostained for pS129- $\alpha$ S and processed for electron microscopy. In neurons the $\mathrm{pS} 129-\alpha \mathrm{S}$-associated gold particles were associated with the ER membranes (arrowheads). No labeling was seen in $n T g$ samples $(\boldsymbol{H}, J)$. The ER cisternae in diseased $A 53 T \alpha S \operatorname{Tg}$ mice were often severely dilated (asterisks) while the ER in $\mathrm{nTg}$ controls showed a normal morphology. Scale bar, $50 \mathrm{~nm}$.

located within the lumen of microsomes and not merely attached to the membrane surface.

Subcellular fractionation of symptomatic $A 53 \mathrm{~T} \alpha \mathrm{S} \mathrm{Tg}$ mice reveals that higher molecular weight $\alpha \mathrm{S}$ and pSer129- $\alpha \mathrm{S}$ are enriched in ER/M fraction (Fig. $4 E, F$ ), indicating that ER could be directly affected by $\alpha \mathrm{S}$ pathology. However, because $\alpha \mathrm{S}$ aggregates can be pelleted by centrifugation, cofractionation of $\alpha S$ with $\mathrm{ER} / \mathrm{M}$ could represent a fortuitous cosedimentation. To control for this possibility, we used the membrane floatation assay (Ding et al., 2002) to determine whether the $\alpha \mathrm{S}$ aggregates "float" with the ER/M membranes on a density gradient. Analysis of the "membrane" and the "free" fractions obtained following the gradient centrifugation of the ER/M preparations from SpC show that both monomer and aggregated $\alpha \mathrm{S}$ were recovered with the membranes along with the ER marker, calnexin (Fig. 4G). Microsomal $\alpha$ S monomers from $A 30 P$ (Fig. $4 G$ ) and $\mathrm{nTg}$ mice (data not shown) are also recovered with the membranes in this assay. In contrast, total Triton X-100-insoluble $\alpha \mathrm{S}$ aggregates (TX- $\mathrm{P}_{\text {tot }}$ ) 

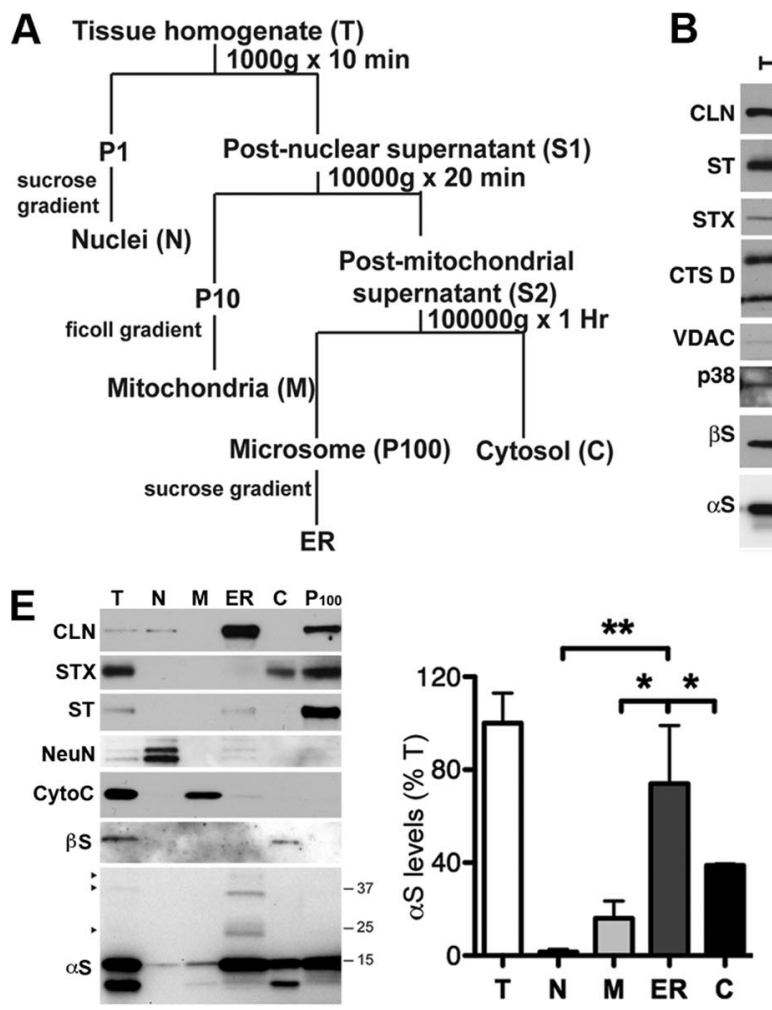
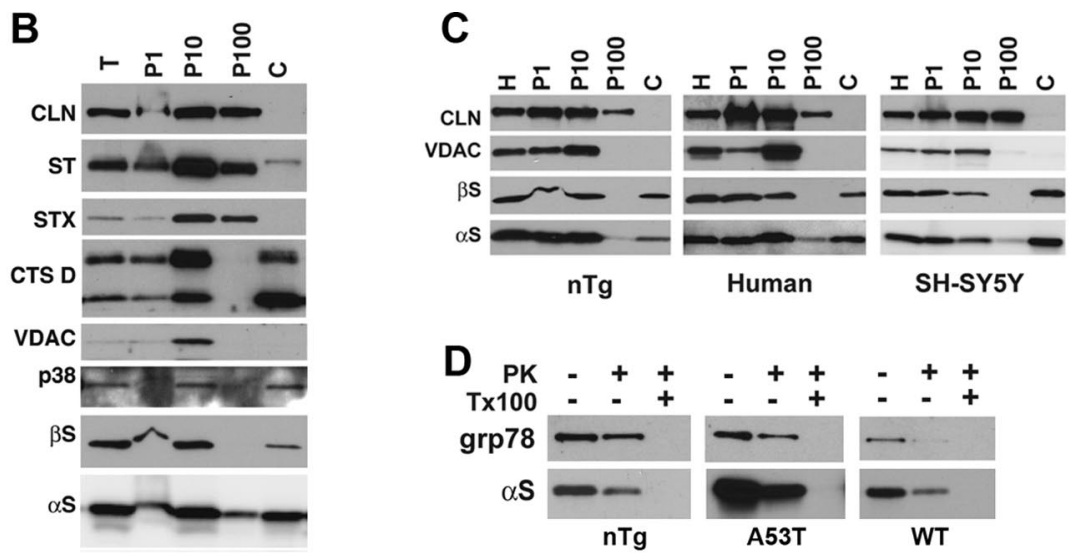

$\mathrm{nTg}$

Human

SH-SY5Y

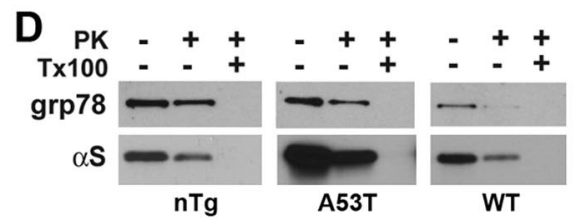

$\mathbf{F}$

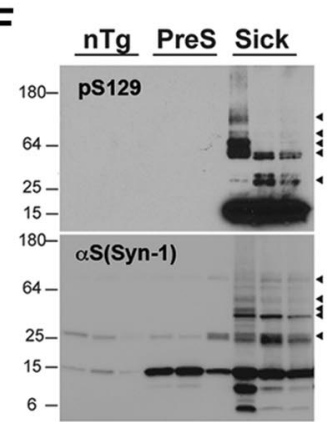

Figure 4. Accumulation and aggregation of $\alpha S$ in the ER. $A$, Schematic representation of the protocol used for subcellular fractionation. $B$, Various subcellular fractions, as shown in ( $A$ ), from presymptomatic $A 53 T \alpha$ S mice were analyzed for organelle markers: calnexin (CLN) for ER, synaptotagmin (ST) for synaptic vesicles, syntaxin 6 (STX) for Golgi, cathepsin D (CTSD) for lysosomes, VDAC for mitochondria, and p38 for cytosol. Parallel analysis for $\alpha S$ and $\beta S$ shows ER-associated $\alpha$ but not $\beta S$. C, $\alpha$ S, but not $\beta S$, accumulates within the microsome fraction. ER/M (P100) and other intermediate fractions from nTg mice, human pons, and SH-SY5Y cell lines stably transfected with $\alpha$ S or $\beta$ S were analyzed for $\alpha S / \beta S$ and organelle markers. In all cases, $\alpha S$, but not $\beta S$ is associated with ER/M fraction. Note that even with the overexpression of $\beta S$ in the SH-SY5Y cell lines, $\beta$ S does not associate with ER/M fractions (P100). D, ER-associated $\alpha S$ monomers in $n T g, A 53 T \alpha S T g$, and WT $\alpha$ S Tg mice are protected from proteolysis by PK, indicating that $\alpha$ S is in the ER lumen. In the presence of $1 \%$ TX-100, both $\alpha$ S and the ER-resident grp78 are completely proteolyzed by PK. Identical results were obtained with $A 30 P \alpha S$ mice. $E$, Accumulation of $\alpha S$ aggregates with ER-enriched microsomes fractions. One microgram of pure organelle fractions were also evaluated using organelle-specific markers: calnexin (CLN) for ER, synaptotagmin (ST) for synaptic vesicles, syntaxin 6 (STX) for Golgi, NeuN for nuclei (N), cytochrome c (CytoC) for mitochondria (M), $\beta$ S for cytosol (C). Total SDS-soluble (T) and initial ER/M (P100) fractions are also shown. The gradient-enriched ER fraction is free from other organelle markers but is enriched for ER markers. The ER fraction also shows significant enrichments in $\alpha S$ monomer and the presence of SDS-stable high molecular weight $\alpha$ S aggregates (arrowheads). Quantitative data show that $\alpha S$ monomer is significantly enriched in the ER fractions, compared with M and N. All organelles were fractionated from SpCs of end-stage A53T $\alpha S \operatorname{Tg}$ mice. Values are mean \pm SEM $(n=3-4) .{ }^{*} p<0.05$, ${ }^{* *} p<0.01$, one-way ANOVA. F, Microsomes from end-stage $A 53 \mathrm{~T} \alpha \mathrm{S} \mathrm{Tg}$ mice show accumulation of $\mathrm{pS} 129 \alpha \mathrm{S}$ and oligomeric $\alpha \mathrm{S}$ (arrowheads). Equal amounts of microsomes obtained from $\mathrm{SpC}$ of nTg and $A 53 \mathrm{~T} \alpha \mathrm{S} \mathrm{Tg}$ mice were immunoblotted for pS129 $\alpha$ S and total $\alpha$ S. Asymptomatic, (PreS); end stage, (Sick). G, Membrane floatation analysis of microsomes and detergent-insoluble $\alpha$ S. Microsome-associated $\alpha S$ from end-stage $A 53 T \alpha S$ Tg mice (Sick) and age-matched A30P $\alpha$ S Tg mice shows that $\alpha S$ floats with membranes. Lanes are input microsomes (P100), membrane fractions (M), and free (F) fractions from the floatation gradient. The detergent-insoluble $\alpha \mathrm{S}$ aggregates (P-Tx) do not float with the $\mathrm{M}$ fractions. Arrowheads are high molecular weight aggregates of $\alpha \mathrm{S}$.

isolated from $\mathrm{SpC}$ of end-stage $\mathrm{A} 53 \mathrm{~T}$ mice are recovered in the free fraction and not in the membrane fraction (Fig. 4G). Thus, intact membranes are required for $\alpha \mathrm{S}$ aggregates to float on the density gradient. Collectively, our results confirm that significant fraction of $\alpha \mathrm{S}$ aggregates are actively bound to the microsomes.

$\alpha S$ accumulates in ER with disease, binds to ER chaperones, and $\alpha S$ overexpression sensitizes neuronal cells to ERS-induced toxicity

Since both $\alpha \mathrm{S}$ and $\alpha \mathrm{S}$ aggregates associate with ER/M, we asked whether quantitative changes in the microsomal $\alpha \mathrm{S}$ levels correlate with the development of disease in the brain. In presymptomatic $\mathrm{Tg}$ mice, the level of $\mathrm{ER} / \mathrm{M} \alpha \mathrm{S}$ was directly proportional to the total level of $\alpha \mathrm{S}$ expression. Thus, mice from all $\mathrm{Tg}$ lines showed increased levels of ER/M $\alpha \mathrm{S}$ compared with nTg mice (Fig. 5A). However, in the A53T $\alpha S \mathrm{Tg}$ mice, the relative abundance of microsomal $\alpha \mathrm{S}$ increases with disease progression in the pathologically affected areas (SpC, BrSt) (Fig. 5A,B). In particular, while the $\mathrm{BrSt} / \mathrm{SpC}$ express lower levels of total $\alpha \mathrm{S}$ than the cortex (Ctx), the microsomal $\alpha \mathrm{S}$ is significantly higher in $\mathrm{BrSt} /$ $\mathrm{SpC}$ than the Ctx (Fig. 5B). Thus, there is a selective ER/M accumulation of $\alpha \mathrm{S}$ in the areas that are vulnerable to $\alpha$-synucleinopathy. Significantly, the analysis of ER/M fractions prepared from human PD cases also show that the level of microsomal $\alpha \mathrm{S}$ is significantly higher in the PD cases compared with the non-PD controls (Fig. 5C).

Given the presence of $\alpha \mathrm{S}$ and $\alpha \mathrm{S}$ aggregates in the ER lumen, we asked whether $\alpha$ S promotes activation of ERS via competing for binding to ER chaperones. We used the ER/M fractions to determine whether $\alpha \mathrm{S}$ can coimmunoprecipitate grp78 and grp94. Our results show that immunoprecipitation of microsomal $\alpha \mathrm{S}$ also recovers grp78 and grp94 (Fig. 5D). Similarly, immunoprecipitation of grp78 leads to recovery of $\alpha \mathrm{S}$ (Fig. $5 D$ ). Moreover, interaction of $\alpha \mathrm{S}$ with ER chaperones occurs in both asymptomatic and symptomatic Tg mice indicating that ER chaperones normally interact with $\alpha \mathrm{S}$ monomers (Fig. 5D). However, the interaction between $\alpha \mathrm{S}$ and ER chaperones could be favored with an increase in the microsomal $\alpha \mathrm{S}$ levels, as we were not able to consistently demonstrate interaction of endogenous mouse $\alpha \mathrm{S}$ with ER chaperones (Fig. 5D).

If increased microsomal $\alpha \mathrm{S}$ leads to increased interactions between $\alpha \mathrm{S}$ and ER chaperones, increased $\alpha \mathrm{S}$ expression may also 

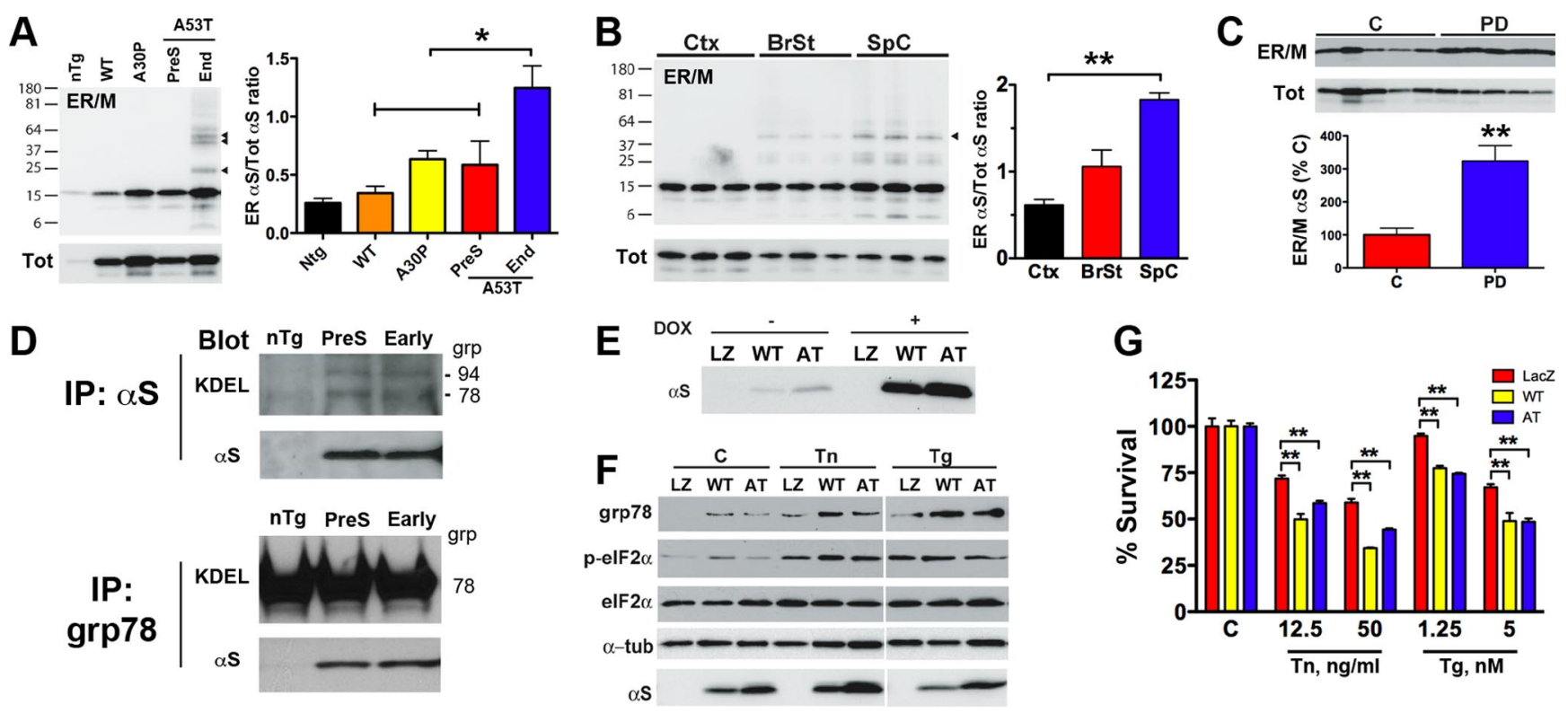

Figure 5. ER/M $\alpha$ S increase with the progression of $\alpha$-synucleinopathy sensitizes neural cells to ERS-induced toxicity. $\boldsymbol{A}$, Immunoblot analysis of $\alpha \mathrm{S}$ levels in microsomes (ER/M) and total SDS-soluble lysates (Tot) obtained from different mouse lines. The samples were obtained from spinal cord. The graph shows the quantitative analysis of the immunoblots where the relative levels of microsomal $\alpha$ S normalized to total $\alpha$ (mean $\pm S D, n=3-4),{ }^{*} p<0.05$, one-way ANOVA. $\boldsymbol{B}$, Immunoblot analysis of $\alpha$ S levels in microsomes (ER/M) and total SDS-soluble lysates (Tot) obtained from different brain regions of end-stage $A 53 T \alpha S \mathrm{Tg}$ mice. The graph shows the relative levels of microsomal $\alpha$ S normalized to total $\alpha S$ (mean \pm SD, $n=3-4)$, ${ }^{* *} p<0.01$ (Ctx vs SpC), one-way ANOVA. C, Immunoblot analysis of $\alpha$ S levels in microsomes (ER/M) and total SDS-soluble lysates (Tot) obtained from pons of human PD cases and controls. The graph shows the levels of microsomal $\alpha$, normalized to the levels in the controls $(C, 100 \%) . n=5,{ }^{* *} p<0.001$, Student's $t$ test. $D, \alpha S$ interacts with ER luminal chaperones grp78 and grp94. Immunoprecipitation with syn-1 antibody (IP: $\alpha$ S) or anti-grp78 antibody (IP:grp78) was performed on purified ER/M from nTg, asymptomatic A53T $\alpha$ S Tg (PreS), and end-stage A53T $\alpha$ S Tg (End) mice. Immunoprecipitates' pellets were probed with antibody for grp78 and grp94 (KDEL) and NAC-1 for $\alpha$ S. E, Doxycyclin (Dox)-dependent induction of $\alpha S$ expression in BE(2)-M17 cell lines. Lanes, LacZ (LZ); WT $\alpha S$ (WT); and A53T $\alpha$ S (AT). $F$, Immunoblot analysis of ER stress markers in M17 cells treated with tunicamycin (Tn) $12.5 \mathrm{ng} / \mathrm{ml}$ or thapsigargin (Tg) $5 \mathrm{~nm}$ for $24 \mathrm{~h}$. Cells were induced for transgene expression for $3 \mathrm{~d}$ before treatment. C, control. G, Inducible $\alpha$ S BE(2)-M17 cells lines were induced for $3 \mathrm{~d}$ before treatment with increasing concentration of $\operatorname{Tn}(12.5$ or $50 \mathrm{ng} / \mathrm{ml})$ or Tg (1.25 or $5 \mathrm{~nm})$ for $24 \mathrm{~h}$. Cell survival was tested with XTT-based colorimetric method. The values are normalized to the control untreated cells and are mean \pm SD $(n=4)$. ${ }^{* *} p<0.001$, two-way ANOVA.

sensitize neuronal cells to ERS-induced toxicity. Such would be significant for age-related vulnerability to $\alpha$-synucleinopathy as increased ERS is likely associated with aging and/or environmental toxin exposure. Thus, we examined if increased expression of $\alpha S$ could affect the vulnerability of neuronal cells to inducers of ERS. We used M17 cell lines that show doxycycline-inducible expression of WT Hu $\alpha$ S, A53T Hu $\alpha$ S, or LacZ (Fig. 5E). Following the transgene induction, the cells were exposed to increasing concentration of ER stressors (tunicamycin or thapsigargin) for $24 \mathrm{~h}$. Analysis of the cells for ERS markers in the M17 cell lines show that, even without exogenous ER stressors, overexpression of $\alpha \mathrm{S}$ is sufficient to cause modest basal activation of grp78. With the exogenous ER stressors, $\alpha \mathrm{S}$ expressing cells show higher induction of grp78 compared with LacZ controls (Fig. 5F). Significantly, analysis of phospho-eIF $2 \alpha$ shows that the basal $\alpha$ Sdependent induction of grp78 is not associated with increased phospho-eIF $2 \alpha$. Moreover, despite the greater induction of grp78 following ER stressor to Hu $\alpha \mathrm{S}$-expressing cells, the levels of phospho-eIF $2 \alpha$ following ERS was comparable in all cells (Fig. $5 F)$. Analysis of cell survival shows that increased expression of either WT or A53T Hu $\alpha$ S leads to increased vulnerability of the M17 cells to ER stressors (Fig. 5G). In addition, we observe that human catecholaminergic neuroblastoma lines (SH-SY5Y) stably expressing $\mathrm{Hu} \alpha \mathrm{S}$ (Li and Lee, 2005) are also more sensitive to cell death caused by ER stressors (data not shown). These results show that in cultured cell lines, overexpression of either WT or mutant $\alpha \mathrm{S}$ can reliably cause modest levels of ERS and sensitizes cells to ER stress. Combined with the induction of $\alpha \mathrm{S}$ pathology, as with the expression of A53T mutant, in vivo, ER/M-associated $\alpha S$ likely contributes to neurodegeneration.

\section{Activation of ER caspase and ER accumulation of polyubiquitin in $A 53 T \alpha S \mathrm{Tg}$ mice}

The above results indicate that $\alpha$-synucleinopathy in $A 53 \mathrm{~T} \alpha S \mathrm{Tg}$ mice is associated with chronic ERS and overexpression of $\alpha \mathrm{S}$ sensitizes neural cells to ER stressors. Combined with the presence of abnormal ER morphology and lack of increase in phospho-eIF $2 \alpha$, the conditions in the mice could promote the activation of cell death pathways. Thus, we examined whether the activation of ERS-associated caspase activation, such as cleavage/activation of caspase-12 in rodents (Zhang and Kaufman, 2006), occurs in the diseased $A 53 T \alpha S \operatorname{Tg}$ mice. Our analysis shows that $\alpha$-synucleinopathy is associated with the increased cleavage of caspase-12 and other downstream caspases (Fig. $6 \mathrm{~A}$ ). The activation of caspase- 12 is selective for $\alpha$-synucleinopathy since analyses of presymptomatic and pathology-free region (cortex) do not show accumulation of caspase-12 (Fig. 6B,C).

Previous studies indicate that overexpression of $\alpha \mathrm{S}$ can cause ubiquitin-proteasome system (UPS) stress (Smith et al., 2005), and proteasome inhibition can cause abnormal UPR characterized by attenuated PERK-dependent phosphorylation of eIF2 $\alpha$ (Nawrocki et al., 2005). Thus, we asked if $\alpha$-synucleinopathy in mice was associated with signs of UPS stress (i.e., accumulation of polyubiquitinated proteins) to the ER. Analyses of unfractionated $\mathrm{SpC}$ extracts show that the disease in the symptomatic $A 53 T \alpha S \mathrm{Tg}$ mice is associated with moderate $(\sim 2$ - to 3 -fold $)$ increase in the levels of polyubiquitin in various extracts (Fig. $6 D$ ). However, when the ER/M fractions were analyzed for the polyubiquitin levels, ER/M from the symptomatic $A 53 \mathrm{~T} \alpha \mathrm{S} \mathrm{Tg}$ mice showed a more dramatic increase ( $\sim 20$ - to 40 -fold) in the polyubiquitin levels (Fig. 6E). Moreover, parallel analyses of 

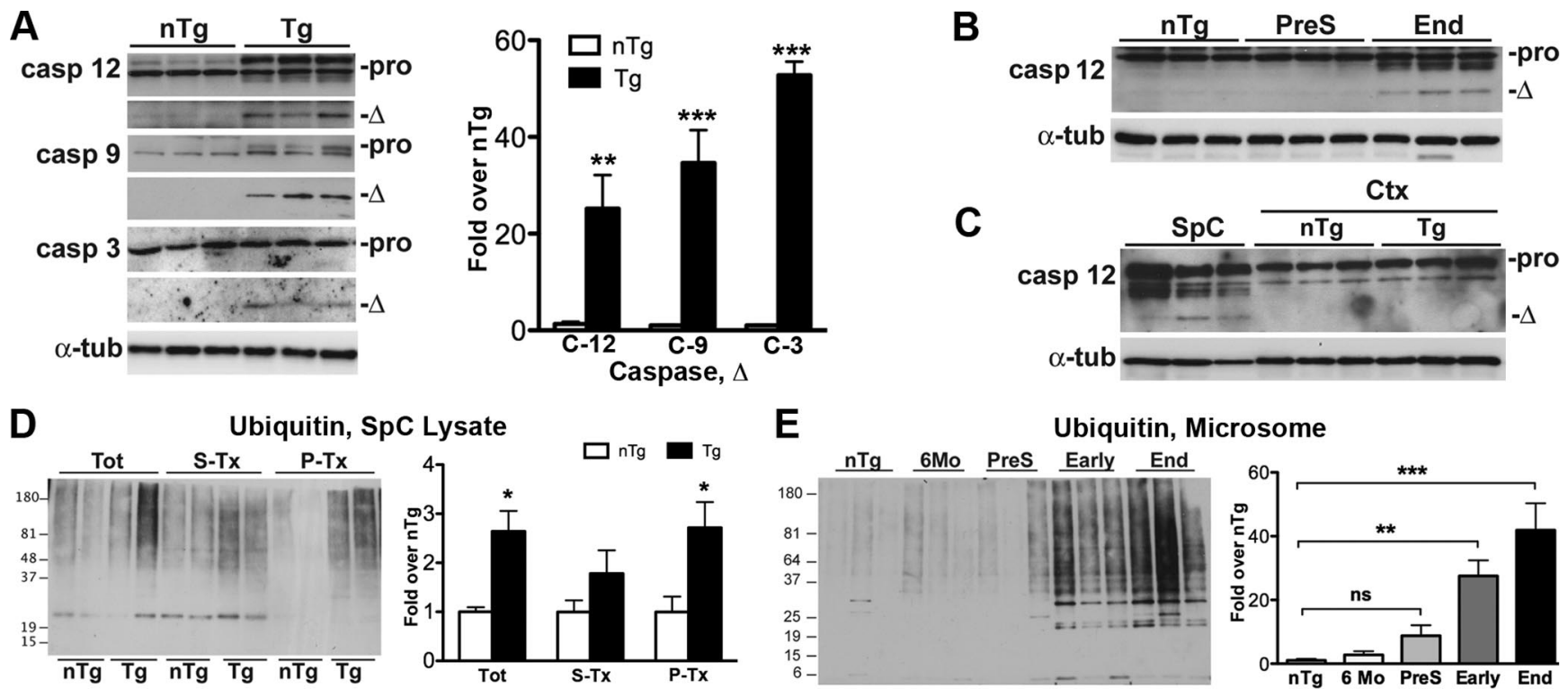

E

Ubiquitin, Microsome
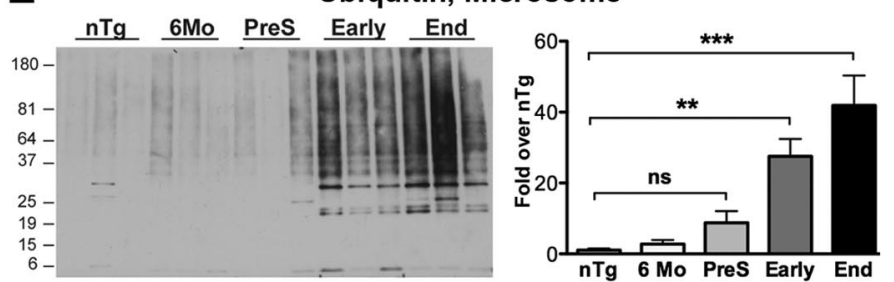

Figure 6. ER accumulation of polyubiquitin, and ER caspase activation with $\alpha$-synucleinopathy in the $A 53 T \alpha S \operatorname{Tg}$ mice. A, Immunoblot analysis of total SpC lysates from the end-stage $A 53 T \alpha S$ $\mathrm{Tg}$ mice and $\mathrm{nTg}$ littermates. Neuropathology in the A53T $\alpha$ S Tg mice is clearly associated with cleavage of ER-dependent caspase- 12 and its downstream effectors, caspase- 9 and -3 (pro, pro-peptide; $\Delta$, cleaved). Both pro-peptide and cleaved peptide were detected on the same blot but cropped to save space. The bar graph is the quantitative analysis of blots shown. The amount of cleaved products was normalized to the full-length protein and represents mean $\pm \operatorname{SEM}(n=4) .{ }^{* *} p<0.001,{ }^{* *} p<0.01$, Student's $t$ test, Tg versus $n T g$ littermates. $B, C$, Caspase- 12 activation is selective for $\alpha$-synucleinopathy. (B) Caspase-12 immunoblot showing cleavage ( $\Delta$ ) of caspase-12 propeptide (pro) in end-stage (End) Tg mice compared with presymptomatic (PreS) mice. ( $\boldsymbol{C}$ ) Caspase-12 analysis of affected areas $(\mathrm{SpC})$ compared with disease-free regions (Ctx) from end-stage $\mathrm{Tg}$ and $\mathrm{nTg}$ mice. Note that the cleaved caspase is associated with the presence of end-stage disease. D, Ubiquitin immunoblot of total SDS-soluble fraction (Tot), Triton X-100-soluble (S-Tx), and Triton X-100-insoluble pellets (P-Tx) from SpC of end-stage A53T $\alpha S$ Tg mice and nTg littermates. The graph shows quantitative analysis. Mean \pm SEM $(n=6),{ }^{*} p<0.05$, Student's $t$ test. $E$, Ubiquitin immunoblot of microsome fractions from $n T g$ and $A 53 T \alpha S T g$ mice at various stages: 6 months old, 10 months old, presymptomatic stage (PreS), and symptomatic stages (Early, End). Densitometry analysis of immunoblots reveals the dramatic ER accumulation (30- to 40 -fold increase) of polyubiquitin with $\alpha$-synucleinopathy. In contrast, total tissue increase in polyubiquitin is only 2-to 3 -fold (D). The plots are mean \pm SEM of percentage of $n T g$ average $(n=6)$. ${ }^{* * *} p<$ $0.001,{ }^{* *} p<0.01$, one-way ANOVA. Both Early- and End-stage Tg mice are also different from PreS $(p<0.05$ and $p<0.001)$.

ER/M from $A 53 T \alpha S$ Tg mice at different disease stages show a progressive increase in polyubiquitin levels with the disease progression (Fig. 6E). These results suggest dysfunctional ER and abnormal ER-associated protein degradation (ERAD) with $\alpha$-synucleinopathy. While additional studies are required to fully evaluate ERAD and UPS stress in $\alpha$-synucleinopathy, our results suggest that $\alpha$-synucleinopathy is associated with multiple markers of ER dysfunction.

\section{Anti-ERS compound, Salubrinal, extends life span and reduces ER accumulation of $\alpha S$ in $A 53 T \alpha S T g$ mouse model of $\alpha$-synucleinopathy}

The above studies show there are temporal and spatial associations between $\alpha \mathrm{S}$ abnormalities, chronic UPR, and neurodegeneration. However, it will be important to show if the aspects of $\alpha$-synucleinopathy-linked chronic ERS documented here are mechanistically linked to the onset and/or progression of $\alpha$-synucleinopathy in vivo.

To determine whether chronic ERS-related toxicity is mechanistically linked to the onset and/or progression of disease in vivo, we treated cohorts of $A 53 T \alpha S$ Tg mice (line G2-3) with Salubrinal, a compound known to protect cells from chronic ER stress by inhibiting dephosphorylation of eIF2 $\alpha$ (Boyce et al., 2005). Salubrinal has been shown to partially attenuate PC12 cells from A53T $\alpha$ S-dependent toxicity (Smith et al., 2005) and to extend the life span of G93A-SOD1 Tg mouse model of motor neuron disease by $\sim 20 \mathrm{~d}(\sim 20 \%)$ (Saxena et al., 2009). Thus, if chronic ER stress is an active and necessary participant in $\alpha$-synucleinopathy, Salubrinal could attenuate the disease manifestation in mice. Moreover, the lack of p-eIF $2 \alpha$ induction in the A53T $\alpha$ S Tg mouse model provides further rationale for using
Salubrinal. Since the $A 53 T \alpha S$ Tg mouse model used here is one of the few models of fatal $\alpha$-synucleinopathy, as it would be in humans, the life span was used as the primary outcome measure for the potential therapeutic effects of Salubrinal on $\alpha$-synucleinopathy. To minimize any adaptive effects of treatment, Salubrinal treatment was initiated at 12 months of age. At this age, $\sim 20 \%$ of $A 53 T \alpha S$ Tg mice cohorts in various groups had developed the disease at the same rate (Fig. 7A). However, following the initiation of Salubrinal treatment, the rate of disease onset in the Salubrinal group was obviously slower than the control cohort (Fig. 7A).

Analysis of brain extracts from Salubrinal- and vehicle-treated mice shows that while Salubrinal treatment did not consistently lead to increase in p-eIF $2 \alpha$ levels (Fig. $7 B, C$ ), there was consistent and significant increase in the levels of $\mathrm{CHOP}$ expression (Fig. $7 B, C$ ), a downstream reporter of p-eIF2 $\alpha$ (Ron and Walter, 2007).

To determine whether the Salubrinal treatment directly impacts $\alpha \mathrm{S}$ expression or development of $\alpha \mathrm{S}$ abnormalities, the brain lysates were analyzed for $\alpha$ S levels. The results show that the levels of total SDS-soluble $\alpha \mathrm{S}$ were not affected by the Salubrinal treatment (Fig. 7D), confirming that Salubrinal did not simply reduce overall $\alpha \mathrm{S}$ expression. However, Salubrinal treatment was associated with significantly reduced microsomal accumulation of monomeric and oligomeric $\alpha \mathrm{S}$ (Fig. 7D). Moreover, our companion analysis for toxic $\alpha \mathrm{S}$ oligomers shows that Salubrinal treatment attenuates the accumulation of toxic $\alpha \mathrm{S}$ oligomers (Colla et al., 2012).

While Salubrinal treatment delayed the onset of motoric symptoms, Salubrinal treatment did not attenuate the progression of the disease following onset [16.7 $\pm 3.4 \mathrm{~d}$ (vehicle) compared with $17.4 \pm$ 
A

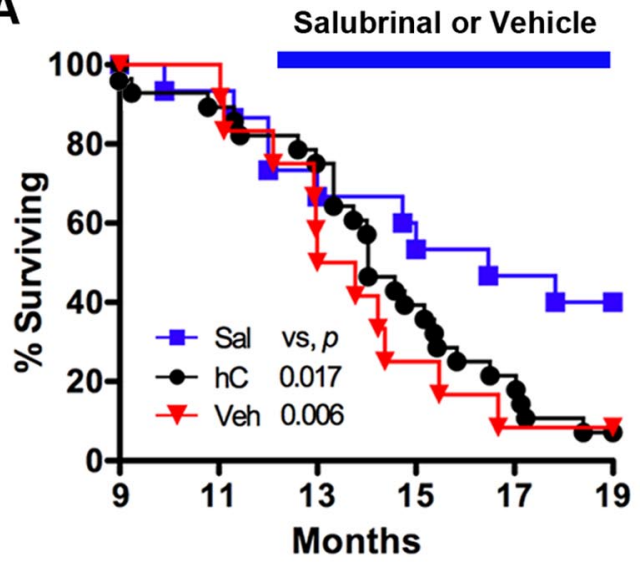

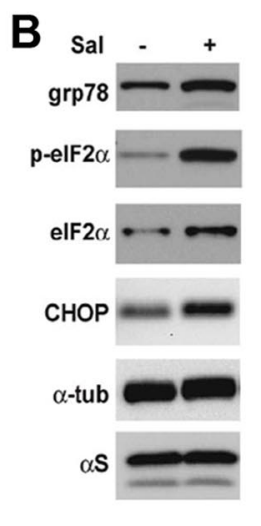

D
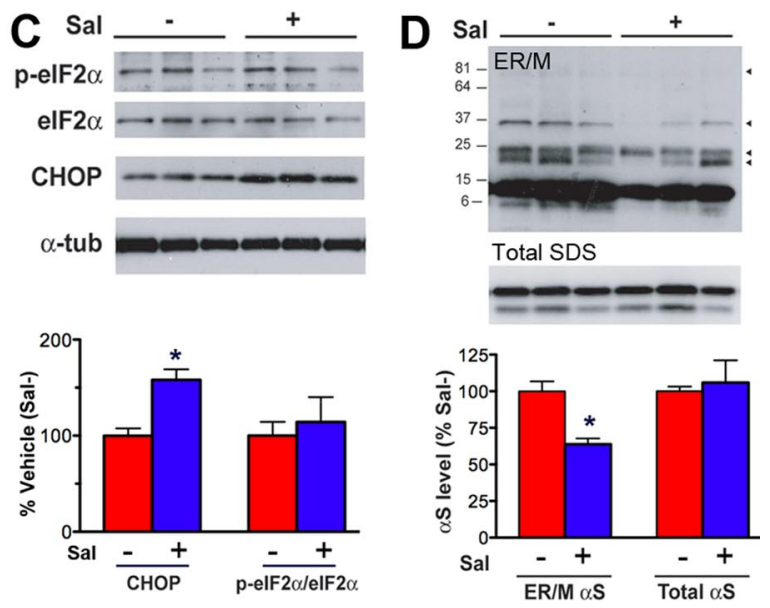

Figure 7. Salubrinal delays the onset of motoric dysfunction in the A53T $\alpha$ STg mouse model of $\alpha$-synucleinopathy. $A$, Survival curve of $A 53 T \alpha S$ mice (line $62-3$ ) treated with Salubrinal (Sal, $n=$ 11) or vehicle (Veh, $n=10$ ) and historical controls ( $h C, n=23$ ) show that Salubrinal delays the disease manifestation in the A53T $\alpha$ S mice ( $p<0.01$, Sal vs Veh; $p<0.05$, Sal vs $h C ;$ not significant, $h C$ vs Veh; log-rank test). The hC represents the life span of all A53T $\alpha$ S mice followed in the lab within the 1 year before treatment. The survival fractions between the hC and Veh mice were comparable, indicating that mouse handling did not influence their life span. The bar indicates the duration of the Salubrinal or vehicle treatments. $\boldsymbol{B}, \boldsymbol{C}, \mathbf{I m m u n o b l o t}$ analysis of total $\mathrm{SpC}$ lysates from the end-stage $A 53 T \alpha S$ mice treated with the vehicle (Sal-) or Salubrinal (Sal+). Among the four Salubrinal-treated mice analyzed, one mice showed induction of p-elF2 $\alpha(\boldsymbol{B})$. In other mice, Salubrinal treatment was only associated with the accumulation of CHOP, a downstream reporter for p-elF2 $\alpha$ (C). The densitometric analysis of immunoblots shows induction of CHOP. Values (mean \pm SEM) are the percentage of average Sal - levels $(n=3) .{ }^{*} p<0.05$, Student's $t$ test. $D, \alpha$ S immunoblot of microsomal proteins (ER/M) and the total SDS-soluble proteins from SpC of vehicle (Sal-)- and Salubrinal (Sal+)-treated A53T $\alpha$ S mice. Arrowheads are high molecular weight aggregates of $\alpha S$. The graph shows the quantitative analysis of the immunoblots. The ER/M fractions from Salubrinal (Sal)-treated A53T $\alpha$ S mice shows a significant decrease in $\alpha S$ while total $\alpha S$ is unchanged. Values are mean \pm SEM $(n=3)$, ${ }^{*} p<0.05$, Student's $t$ test.

$2.7 \mathrm{~d}$ (Salubrinal)]. Thus, immunocytochemical analysis of end-stage Tg mice for the accumulation of pSer129- $\alpha \mathrm{S}$ or other neuropathology did not reveal obvious differences between the Salubrinal- and vehicle-treated mice. Thus, Salubrinal may provide protection from $\alpha$-synucleinopathy by selectively decreasing the ER accumulation of $\alpha \mathrm{S}$ and $\alpha \mathrm{S}$ oligomers. This result also supports the view that microsomal $\alpha \mathrm{S}$ oligomers are of pathologic importance. Moreover, our results suggest that anti-ER stress compounds, such as Salubrinal, should be developed as a therapy for $\alpha$-synucleinopathy.

\section{Salubrinal attenuates disease manifestation and decreases Golgi fragmentation in the AAV-rat model of A53T $\alpha$ S-dependent DAergic neurodegeneration}

Because $A 53 T \alpha S$ Tg mice lack robust DAergic pathology, we used an AAV-transduced rat model to ask whether Salubrinal could also attenuate DAergic neurodegeneration following the overexpression of A53T Hu $\alpha$ S in rat SNpc DA neurons. Unilateral injections of the $A A V 2 / 6-p g k-\alpha S-A 53 T-W P R E$ vector in the rat $S N p c$ achieve widespread expression of $H u \alpha S$ in DA neurons and a progressive degeneration of $S N p c$ neurons (Kirik et al., 2002; Lo Bianco et al., 2002; Koprich et al., 2010) (Fig. 8 A). To investigate whether Salubrinal protects neurons from A53T $\alpha$ S-induced neurodegeneration, the rats were administered either Salubrinal or vehicle starting at 1 week post $A A V$ injection and evaluated at 12 weeks post $A A V$ injection. Initial immunocytochemical analysis shows that Salubrinal treatment did not have an obvious, if any, effect on the expression of Hu $\alpha \mathrm{S}$ in SNpc (Fig. 8B). During the treatment, the animals were monitored for apomorphine-induced rotational behavior and spontaneous motor asymmetry (Fig. 8C,D).

The $A 53 T \alpha S$ vector-injected rats progressively developed signs of asymmetric motor behavior. In the cylinder test, the left paw contralateral to the injected $S N p c$ was persistently impaired at both $6(23.7 \pm 2.4 \%$ of total touches $)$ and 12 weeks $(18.7 \pm$ $7.1 \%$ ) post injection (Fig. $8 C$ ). Salubrinal administration significantly attenuated the progression of the motor deficit, particularly at 6 weeks $(35.6 \% \pm 3.7 \%$ of total, $p<0.05)$ following injection. Measurement of apomorphine-induced rotations at 12 weeks post injection revealed a similar attenuation of motor abnormalities by Salubrinal (Fig. $8 D$ ). Specifically, while the vehicle-treated, $A 53 T \alpha S$ vector-injected rats showed significant rotational bias compared with the control rats, Salubrinal-treated rats were not significantly different from the controls (Fig. 8D). However, comparisons of Salubrinal- and vehicle-treated groups did not reach statistical significance.

While Salubrinal attenuated the progressive motor abnormalities, the behavioral amelioration by Salubrinal treatment is not reflected in the attenuation of DAergic neurodegeneration (Fig. $8 E, F)$. This raises the possibility that Salubrinal treatment does not prevent the death of DA neurons, but allows remaining neurons to be more functional. To examine this issue, we evaluated the integrity of Golgi apparatus in DA neurons. Fragmentation of the Golgi apparatus is considered an early event preceding neuronal death in response to ER stress (Nakagomi et al., 2008) and has been reported to occur in vivo in conditions of $\alpha \mathrm{S}$ expression (Gosavi et al., 2002). Thus, we hypothesized that Golgi fragmentation could provide a sensitive marker of A53T $\alpha$ S-induced ERstress/toxicity in DA neurons, and may reveal the protective effects of Salubrinal treatment. We performed analysis of Golgi morphology in the DA neurons of the SNpc at 12 weeks post injection using the cis-Golgi matrix protein marker GM130 (Fig. $9 A$ ). Based on the morphology of GM130-positive structures, neurons were classified as "normal" or "fragmented" (Fig. 9A). In the animals injected with the control vector, very little Golgi fragmentation was seen $(\sim 0.9 \%)$ with $>97 \%$ being normal (Fig. $9 B, C)$. The expression of $\mathrm{A} 53 \mathrm{~T} \alpha \mathrm{S}$ led to fragmented and normal Golgi in $16.5 \pm 1.0 \%$ and $67.3 \pm 4.1 \%$ of DA neurons, respectively (Fig. 9B, C). Remarkably, the Salubrinal treatment significantly reduced the proportion of DA neurons with a fragmented Golgi to $4.9 \pm 0.6 \%$ and increased the number DA neurons with a normal Golgi to $85.9 \pm 2.7 \%$ (Fig. 9B, C). Remaining neurons ( $\sim 16 \%$ in Vehicle group and $\sim 10 \%$ in Salubrinal group) were at intermediate states of Golgi morphology and were not classified.

These results indicate that $\mathrm{A} 53 \mathrm{~T} \alpha \mathrm{S}$ toxicity involves disruption of Golgi morphology in the surviving DA neurons at 12 
A
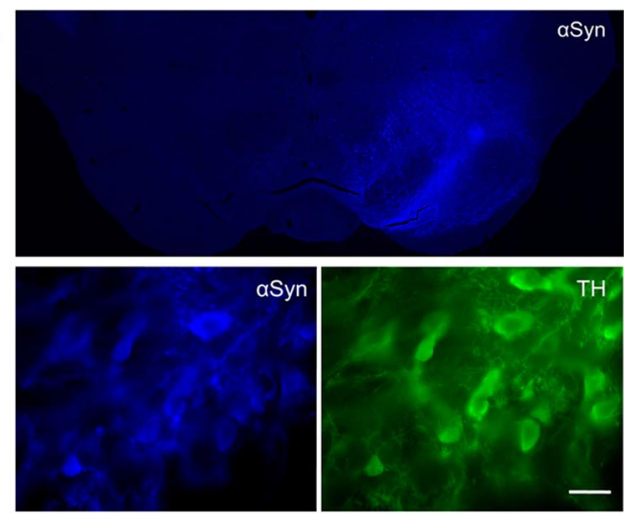

C

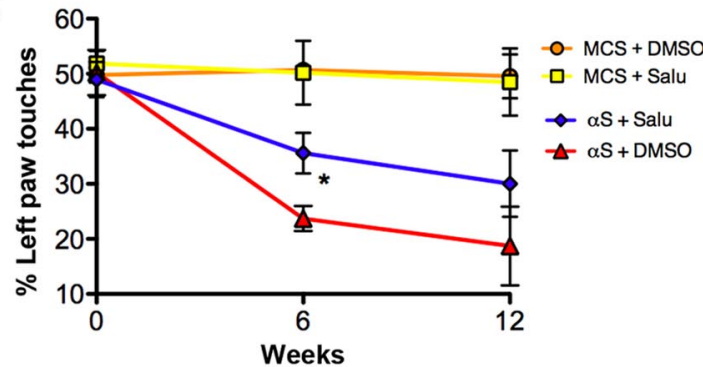

$\mathbf{E}$
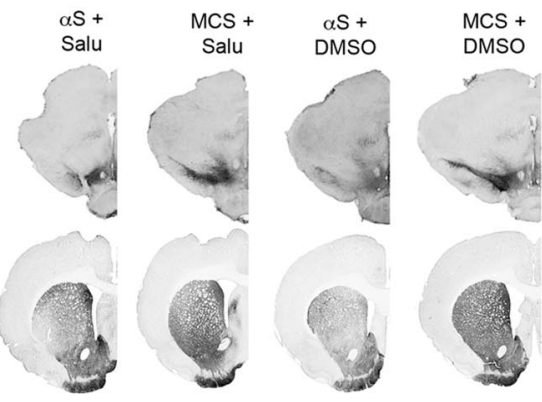

B
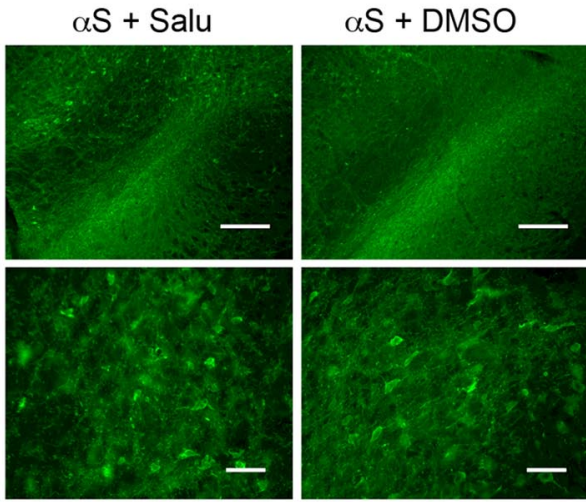

D

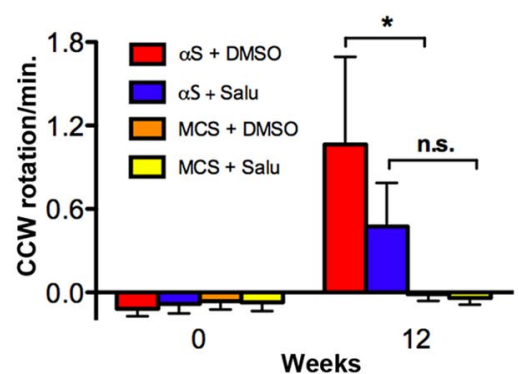

$\mathbf{F}$

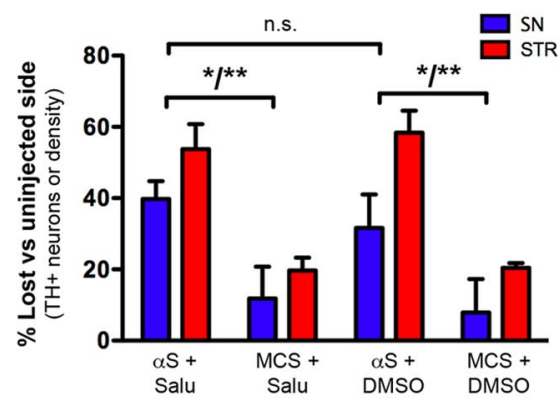

Figure 8. Salubrinal attenuates disease manifestation without protecting from neuronal loss in the $A 53 T \alpha S A A V$ model of DAergic neurodegeneration. $A, A A V-p g k-\alpha S$-A53T stereotactic injection leads to overexpression of $\alpha$ Syn-A53T in the DAergic neurons of the SNpc. Shown are a wide-field and a higher magnification image of representative rat midbrain showing the overexpression of $\alpha$ S(A53T) across the SNpc (blue). TH-positive neurons are green. Scale bar, $10 \mu \mathrm{m}$. B, Salubrinal treatment does not change the level of $\alpha$ S overexpression in the SNpc of rats. Top, Representative low-magnification images of the injected rat SNp c showing $\alpha$ S overexpression in animals treated with either Salubrinal or vehicle (DMSO). Bottom, Representative high-magnification images of nigral neurons showing the overexpression of $\alpha$ S. Scale bars: $500 \mu \mathrm{m}$ (top); $100 \mu \mathrm{m}$ (bottom). C, D, Spontaneous motor behavior measured by forepaw touches in the cylinder test (C) and apomorphine-induced rotational behavior (D). $n=7$ for all groups except the $\alpha S y n+D M S O$ group $(n=6)$; one-way ANOVA followed by Newman-Keuls post hoc test; ${ }^{*} p<0.05$. ns, not significant. $\boldsymbol{E}$, Representative sections of the SNpc (top), and striatum (bottom) immunostained for TH and illustrating nigrostriatal degeneration in all tested experimental conditions. $\boldsymbol{F}$, Stereological analysis reveals that Salubrinal administration does not reduce the significant loss of TH-positive neurons (SN). Similarly, densitometric analysis does not reveal any change in the loss of TH immunoreactivity in the striatum (STR) induced by $\alpha$ S-A53T. Results are expressed as the percentage of loss of SNpc TH-positive neurons or TH immunoreactivity, when compared with the noninjected side, 12 weeks after injection. $n=7$ for all groups except the $\alpha$ Syn + DMSO group $(n=6)$; one-way ANOVA followed by Fisher LSD post hoc test, ${ }^{*} p<0.05$, ${ }^{* * *} p<0.001$.
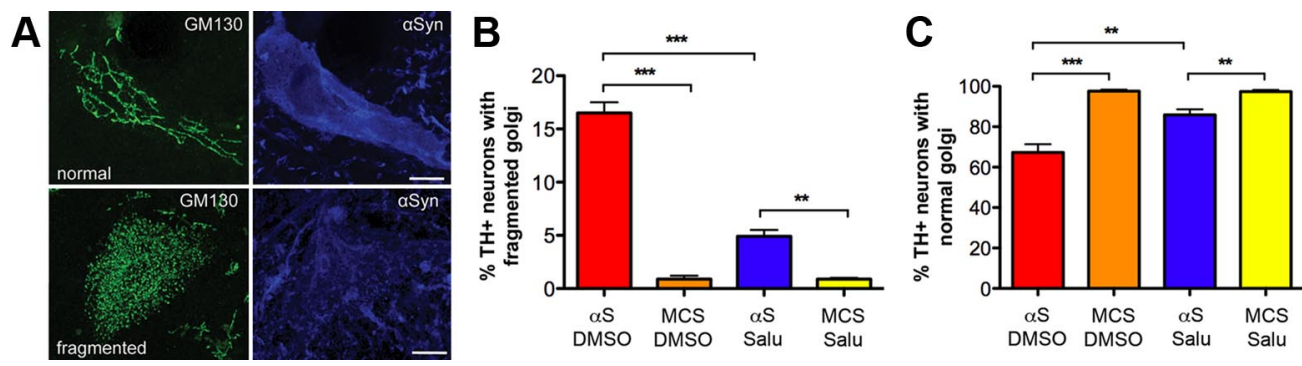

Figure 9. Salubrinal decreases Golgi fragmentation in the A53T $\alpha$ SAAV model DAergic neurodegeneration. $A$, Confocal images of Golgi apparatus (GM130 staining in green) in $\alpha$ S overexpressing neurons ( $\alpha S$ staining in blue) in the rat $S N p$. A well organized network of fine tubules around the nucleus was considered as "normal," while a fine punctuated distribution of the GM130 staining was defined as "fragmented." Other neurons belong to various intermediate stages of fragmentation. B, C, Analysis of Golgi morphology in DA neurons 12 weeks following AAV injection. Salubrinal administration significantly reduces fraction of neurons with fragmented Golgi ( $\boldsymbol{B}$ ) and increases DA neurons with normal Golgi apparatus ( $\boldsymbol{C}$. Thus, Salubrinal protects the Golgi apparatus in nigral DAergic neurons from $\alpha$ Syn-A53T-induced fragmentation. $n=3$ per group, $>400$ cells/animal; one-way ANOVA followed by Tukey's post hoc test, ${ }^{* *} p<0.01,{ }^{* * *} p<0.001$. Scale bar, $5 \mu$ m. 


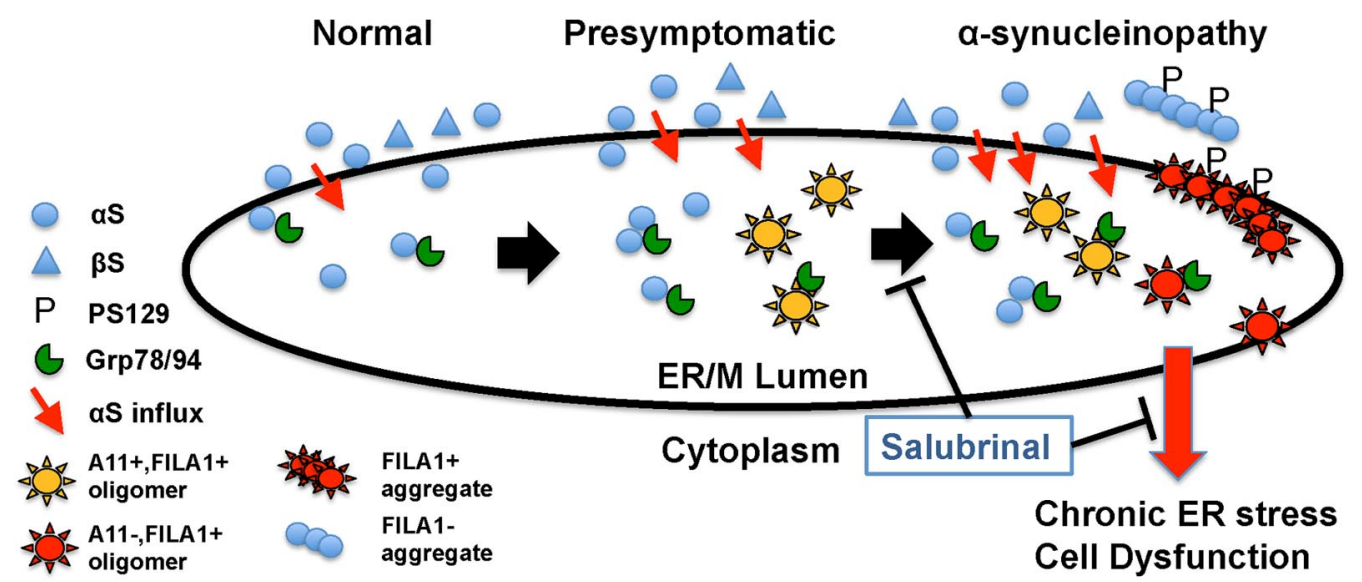

Figure 10. A model showing the evolution of $\alpha \mathrm{S}$ abnormalities in the ER. The model is derived from considering the findings of this report and the companion report (Colla et al., 2012). The red arrows indicating " $\alpha$ flux" are meant to show increased ER accumulation of $\alpha$ S with disease, but the mechanism of $\alpha S$ accumulation in the ER is unknown. Similarly, while our results show Salubrinal inhibits accumulation of $\alpha$ S and reduces ERS, the detailed mechanism is undefined.

weeks post $A A V$ injection, and the Salubrinal treatment attenuates the Golgi fragmentation in surviving DA neurons. However, Salubrinal cannot prevent the initial loss of DA neurons caused by $\mathrm{A} 53 \mathrm{~T} \alpha \mathrm{S}$. This latter fact is not surprising as $\mathrm{A} 53 \mathrm{~T} \alpha \mathrm{S}$, when expressed at sufficient levels, can activate multiple cell death pathways (Smith et al., 2005; Martin et al., 2006).

\section{Discussion}

Our results provide the first direct evidence for pathological involvement of chronic ERS in a vertebrate model of $\alpha$-synucleinopathy. Moreover, we show that UPR associated with $\alpha$-synucleinopathy in brain is abnormal since the induction of ER chaperones is not accompanied by the increase in p-eIF $2 \alpha$. The onset of ERS and disease in the $A 53 T \alpha S$ Tg mice coincides with the accumulation of aggregated $\alpha \mathrm{S}$ with ER microsomes and ERAD defect. More important, activation of ER-associated caspases and attenuation of disease manifestations by ER stress-protective compound, Salubrinal, demonstrate that chronic ERS is an active participant in onset/progression of $\alpha$-synucleinopathy. Our results indicate that reducing the chronic ERS may represent an important disease-modifying therapeutic approach for PD and other $\alpha$-synucleinopathies.

Based on the current results together with our companion report showing the genesis and evolution of toxic $\alpha \mathrm{S}$ oligomers within the ER, we propose a model (Fig. 10) where a small fraction of $\alpha \mathrm{S}$ normally locates to the lumen of the ER/M compartment. With aging and other conditions, $\alpha \mathrm{S}$ oligomer forms and matures into insoluble aggregates with the disease progression. Accumulation and maturation of $\alpha \mathrm{S}$ oligomer is favored by the lack of $\beta S$ in the ER as well as sequestration of ER chaperones by increasing amounts of $\alpha \mathrm{S}$. Initially, soluble $\alpha \mathrm{S}$ monomer and oligomer are not exposed to the cytosol, but the insoluble aggregates become exposed to the cytosol likely by destabilizing the membranes. Together with the fact that therapeutic effects of Salubrinal treatment seem to be associated with reduced $\alpha \mathrm{S}$ oligomers in the ER/M, we hypothesize that the $\alpha \mathrm{S}$-related abnormalities contribute to chronic ERS and neurodegeneration.

Normal presence of $\alpha \mathrm{S}$ in the lumen of ER/M seems consistent with prior data from cell culture showing that $\alpha \mathrm{S}$ can be secreted by neurons by transiting through the normal ER-Golgi secretory compartments (Lee et al., 2005). More recently, Desplats et al. (2009) showed that secreted $\alpha \mathrm{S}$ is transmitted from neuron to neuron, seeding the formation of "aggregates" in the neighbor “accepting" neurons (Desplats et al., 2009). Moreover, recent studies show that secreted $\alpha \mathrm{S}$ can be toxic to neuronal cells (Emmanouilidou et al., 2010). Thus, the presence of toxic $\alpha \mathrm{S}$ species, like the ER-associated $\alpha \mathrm{S}$ aggregates, could be responsible for accelerated inclusion formation in neighboring neurons or neurotoxicity. While the biology behind ER-associated $\alpha \mathrm{S}$ needs further studies, the lack of $\beta S$ in ER/M is a strong indication that ER-associated $\alpha \mathrm{S}$ is not a nonspecific byproduct, lipid-binding, or contamination of subcellular fractionation. Regardless, the fact is that increases in the levels of ER-associated $\alpha \mathrm{S}$ are a common feature of $\alpha$-synucleinopathy in mice and in human support the pathological link between ER-associated $\alpha \mathrm{S}$ and neurodegeneration. Significantly, in addition to $\alpha \mathrm{S}$ monomers, significant amounts of $\alpha \mathrm{S}$ aggregates were selectively associated with microsomes, and our companion report shows that toxic $\alpha$ S oligomers initially form within the ER/M lumen (Colla et al., 2012). Since the ERS response is activated by accumulation of misfolded proteins in the ER (Kozutsumi et al., 1988; Bertolotti et al., 2000) toxic $\alpha$ S oligomers could induce ERS directly by interaction with ER chaperones and/or by affecting ER function (i.e., interfering with calcium metabolism). Even in the absence of $\alpha \mathrm{S}$ aggregate, interactions between $\alpha \mathrm{S}$ and ER chaperones are significant as they could reduce the amount of ER chaperones available to binding to other UPR transducers and ER clients, particularly during ERS conditions. For example, lack of grp78 binding to $\mathrm{IP}_{3}$ Rs could lead to defective $\mathrm{Ca}^{2+}$ homeostasis and mitochondrial defect (Higo et al., 2010). Such conditions are consistent with our data showing that $\alpha \mathrm{S}$ sensitizes cells to ERS toxicity. This finding is significant, as $\alpha \mathrm{S}$ would increase the vulnerability of neurons to ERS caused by aging and/or environmental agents.

The fact that $\alpha$-synucleinopathy in vivo is associated with chronic ERS is consistent with the studies from cellular and invertebrate models that implicate ER stress in $\alpha$ S toxicity (Smith et al., 2005; Cooper et al., 2006). While some of the studies, particularly derived from the yeast model of $\alpha \mathrm{S}$ toxicity (Cooper et al., 2006), suggested that $\alpha S$ monomers could cause ER stress by affecting ER-Golgi membrane trafficking, the ER stress in the vertebrate brain seems mechanistically variable from the $\alpha \mathrm{S}$ induced ER stress in yeast. In yeast, the membrane-binding capacity of $\alpha \mathrm{S}$ monomers seems important for toxicity (Volles and Lansbury, 2007). In brain of $\alpha \mathrm{S}$ Tg mice, ER stress is most obvious with the overt $\alpha \mathrm{S}$ pathology, including ER accumulation of $\alpha \mathrm{S}$ oligomers. However, while $\alpha \mathrm{S}$ toxicity in many viral models is 
independent of $\alpha \mathrm{S}$ membrane-binding properties (Kirik et al., 2002; Lo Bianco et al., 2002; Koprich et al., 2010), toxicity in the rat $A A V 2 / 6$ model is somewhat dependent on membranebinding capacity of $\alpha \mathrm{S}$ (i.e., A30P mutant is least toxic; M. Gaugler and B. Schneider et al., unpublished observation). These variations amplify the notion that multiple toxic pathways are activated by $\alpha \mathrm{S}$ abnormalities, where the mode of toxicity may depend on the context and dynamics of $\alpha S$ expression. In particular, significant early $\alpha \mathrm{S}$ pathology in synaptic terminals and axons do not colocalize with markers of ER (Fig. 3) and may be related to distinct toxic events (Volpicelli-Daley et al., 2011). Moreover, while we hypothesize that $\alpha$-synucleinopathy causes abnormal ERS/UPR, which is mechanistically linked to neurodegeneration, we note that our documentation of ERS/UPR is not complete. In particular, due to technical limitations with reagents, we were not able to effectively evaluate the status of other UPR reporters such as ATF6 and PERK. Because induction of the ER chaperones and the $x b p 1$ cleavage can occur via non-UPR mechanisms (Kim et al., 2008), observed lack of p-eIF2 $\alpha$ induction in $\alpha \mathrm{S}$ Tg mice could reflect activation of processes other than ERS/UPR. Despite this caveat, ER accumulations of both $\alpha \mathrm{S}$ oligomers and polyubiquitin support our view that $\alpha \mathrm{S}$ pathology causes ER dysfunction. We believe future targeted studies using models where UPR pathways are genetically altered will provide valuable insights in this area.

While there are variations in the mechanistic details, it is important that both overt $\alpha$-synucleinopathy in the $A 53 \mathrm{~T} \alpha \mathrm{S} \mathrm{Tg}$ model and $\alpha \mathrm{S}$ toxicity in the rat $A A V 2 / 6$ model is associated with ERS response. The pathologic importance of ERS to neurodegeneration is supported by the recent studies showing that chronic ERS condition can lead to neurodegeneration (Higo et al., 2010), and studies implicate ERS in chronic neurodegenerative conditions (Sokka et al., 2007; Lin et al., 2008; Zhu et al., 2008; Saxena et al., 2009; Egawa et al., 2011; Wang et al., 2011). The pathological significance of chronic ER stress in $\alpha$-synucleinopathy is also supported by our result that pharmacological inhibitor of ER stress can extend the life span of the $A 53 T \alpha S T g$ mouse model and attenuate toxicity of $\alpha \mathrm{S}$ in the AAV2/6-transduced rat model. Significantly, we show here (Fig. 7) and in the companion report (Colla et al., 2012) that Salubrinal is able to selectively reduce levels of ER-associated $\alpha \mathrm{S}$ oligomers without affecting total $\alpha \mathrm{S}$ levels. These results indicate that, consistent with the known action of Salubrinal on ER homeostasis, this compound selectively effects ER accumulation of $\alpha \mathrm{S}$ in models of $\alpha$-synucleinopathy. One intriguing possibility is that since p-eIF $2 \alpha$ has been linked to induction of autophagy (Tallóczy et al., 2002; Py et al., 2009), it is possible that Salubrinal may have facilitated the removal of damaged ER via autophagy. It is also important to note that while Salubrinal is generally considered an anti-ERS compound that inhibits p-eIF $2 \alpha$ dephosphorylation, the exact basis for neuroprotection here and in other studies (Sokka et al., 2007; Zhu et al., 2008; Saxena et al., 2009) is unknown. In the current study, despite the accumulation of $\mathrm{CHOP}$ (Fig. $7 C$ ), we were not able to consistently demonstrate Salubrinal-dependent increase in p-eIF2 $\alpha$ levels (Fig. $7 B, C$ ). Thus, while we and others have used Salubrinal to affect the p-eIF2 $\alpha$ levels in vivo (Kim et al., 2008), we cannot rule out the possibility that the neuroprotective effects of Salubrinal are independent of p-eIF $2 \alpha$ or unrelated to ERS, such as inhibiting translation of protein(s) required for cell death. The possible attenuation of $\alpha \mathrm{S}$ expression by Salubrinal seems unlikely based on our results and the prior studies showing that the attenuation of p-eIF $2 \alpha$ dephosphorylation does not cause a general decrease in protein translation (Jiang et al., 2010). Over- all, while our results provide the initial pathological links between $\alpha$-synucleinopathy, ER stress, and $\alpha \mathrm{S}$ oligomers, the mechanistic details will need further examination.

Salubrinal treatment clearly delays the disease onset in the $A 53 T \alpha S T g$ mouse model and attenuates disease manifestation in the $A A V 2 / 6$ model. However, the results from the AAV2/6 model show that Salubrinal does not increase the survival of DA neurons destined for cell death. Based on the motor tests and the analysis of Golgi morphology, it is clear that Salubrinal corrects $\alpha \mathrm{S}$ induced abnormalities in the surviving neurons. The rescue of Golgi morphology indicates positive effects of Salubrinal on the secretory function of DA neurons, which is further confirmed by improved motor activity in treated animals. Significantly, the therapeutic effects of Salubrinal are comparable to the therapeutic effects of Rab1A overexpression in this model (Coune et al., 2011). Therefore, the defects induced by $\alpha \mathrm{S}$ in the surviving neurons are possibly related to impaired vesicle trafficking at the ER-Golgi level, thereby reducing neuronal secretory capacity. It is also possible that the dose of Salubrinal used was not sufficient to overcome the initial toxic effects of high levels of $\alpha \mathrm{S}$ expression achieved in the AAV2/6 model. Thus, either higher Salubrinal dose and/or longer follow-up may have revealed overt neuroprotection. In particular, Golgi fragmentation is an early precursor to cell death and neuronal health (Nakagomi et al., 2008). Thus, given that Salubrinal reduces Golgi fragmentation in $\alpha \mathrm{S}$ expressing neurons, one may expect less ongoing neurodegeneration at later time points. The results are also consistent with the view that $\alpha \mathrm{S}$ toxicity is associated with the activation of multiple cytotoxic pathways (Vila and Przedborski, 2004; Obeso et al., 2010). The presence of additional toxic processes is also consistent with the fact that while Salubrinal delayed the onset of disease in the $A 53 \mathrm{~T} \alpha \mathrm{S} \mathrm{Tg}$ mice, progression of the disease was not affected. Moreover, in the end-stage animals, we did not observe qualitative differences in neuropathology between Salubrinaland vehicle-treated $A 53 T \alpha S$ Tg mice (data not shown). Another caveat is that the increase in CHOP expression resulting from Salubrinal may have antagonized the neuroprotective effects of Salubrinal as CHOP can promote cell death (Silva et al., 2005). However, the pathogenic importance of CHOP in rodent models of PD is unclear, as despite robust CHOP induction, the loss of $\mathrm{CHOP}$ does not protect from neurodegeneration in some cases (Silva et al., 2005).

In summary, combined with our companion report (Colla et al., 2012), our data show that pathogenic $\alpha \mathrm{S}$ oligomers initially accumulate within ER/M fractions, likely causing ER dysfunction and chronic ER stress. Treating the $\alpha \mathrm{S}$ Tg mouse model the rat $A A V 2 / 6$ model of $\alpha$-synucleinopathy with Salubrinal, a pharmacological inhibitor of ER-stress toxicity, dramatically delays the onset of motoric symptoms and decreases accumulation of $\alpha \mathrm{S}$ oligomers in vivo. Thus, our results establish that toxic $\alpha \mathrm{S}$ oligomers accumulate with $\alpha$-synucleinopathy in brain and identify chronic ER stress as the manifestations of toxicity involved the disease progression. Further, our data indicate that modulation of ERS/UPR constitutes an important therapeutic target for PD and other $\alpha$-synucleinopathies.

\section{References}

Bertolotti A, Zhang Y, Herdershot LM, Harding HP, Ron D (2000) Dynamic interaction of BIP and ER stress transducers in the unfolded-protein response. Nat Cell Biol 2:326-332.

Boyce M, Bryant KF, Jousse C, Long K, Harding HP, Scheuner D, Kaufman RJ, Ma D, Coen DM, Ron D, Yuan J (2005) A selective inhibitor of eIF2alpha dephosphorylation protects cells from ER stress. Science 307:935-939. 
Colla E, Jensen PH, Pletnikova O, Troncoso JC, Glabe CG, Lee MK (2012) Accumulation of toxic $\alpha$-synuclein oligomer within endoplasmic reticulum occurs in $\alpha$-synucleinopathy in vivo. J Neurosci, 32:3301-3305.

Cooper AA, Gitler AD, Cashikar A, Haynes CM, Hill KJ, Bhullar B, Liu K, Xu K, Strathearn KE, Liu F, Cao S, Caldwell KA, Caldwell GA, Marsischky G, Kolodner RD, Labaer J, Rochet JC, Bonini NM, Lindquist S (2006) Alpha-synuclein blocks ER-Golgi traffic and Rab1 rescues neuron loss in Parkinson's models. Science 313:324-328.

Coune P, Bensadoun JC, Aebischer P, Schnieder BL (2011) Rab1A Overexpression prevents Golgi apparatus fragmentation and partially corrects motor deficits in an alpha-synuclein based rat model of Parkinson's disease. J Parkinson's Dis 1:373-387.

Cox B, Emili A (2006) Tissue subcellular fractionation and protein extraction for use in mass-spectrometry-based proteomics. Nat Protoc 1:1872-1878.

Croze EM, Morré DJ (1984) Isolation of plasma membrane, Golgi apparatus, and endoplasmic reticulum fractions from single homogenates of mouse liver. J Cell Physiol 119:46-57.

Desplats P, Lee HJ, Bae EJ, Patrick C, Rockenstein E, Crews L, Spencer B, Masliah E, Lee SJ (2009) Inclusion formation and neuronal cell death through neuron-to-neuron transmission of alpha-synuclein. Proc Natl Acad Sci U S A 106:13010-13015.

Ding TT, Lee SJ, Rochet JC, Lansbury PT Jr (2002) Annular alpha-synuclein protofibrils are produced when spherical protofibrils are incubated in solution or bound to brain-derived membranes. Biochemistry 41:10209-10217.

Duda JE, Giasson BI, Chen Q, Gur TL, Hurtig HI, Stern MB, Gollomp SM, Ischiropoulos H, Lee VM, Trojanowski JQ (2000) Widespread nitration of pathological inclusions in neurodegenerative synucleinopathies. Am J Pathol 157:1439-1445.

Dusonchet J, Bensadoun JC, Schneider BL, Aebischer P (2009) Targeted overexpression of the parkin substrate Pael- $\mathrm{R}$ in the nigrostriatal system of adult rats to model Parkinson's disease. Neurobiol Dis 35:32-41.

Egawa N, Yamamoto K, Inoue H, Hikawa R, Nishi K, Mori K, Takahashi R (2011) The endoplasmic reticulum stress sensor, ATF6alpha, protects against neurotoxin-induced dopaminergic neuronal death. J Biol Chem 286:7947-7957.

Emmanouilidou E, Melachroinou K, Roumeliotis T, Garbis SD, Ntzouni M, Margaritis LH, Stefanis L, Vekrellis K (2010) Cell-produced $\alpha$-synuclein is secreted in a calcium-dependent manner by exosomes and impacts neuronal survival. J Neurosci 30:6838-6851.

Fujiwara H, Hasegawa M, Dohmae N, Kawashima A, Masliah E, Goldberg MS, Shen J, Takio K, Iwatsubo T (2002) alpha-Synuclein is phosphorylated in synucleinopathy lesions. Nat Cell Biol 4:160-164.

Gitler AD, Bevis BJ, Shorter J, Strathearn KE, Hamamichi S, Su LJ, Caldwell KA, Caldwell GA, Rochet JC, McCaffery JM, Barlowe C, Lindquist S (2008) The Parkinson's disease protein alpha-synuclein disrupts cellular Rab homeostasis. Proc Natl Acad Sci U S A 105:145-150.

Gosavi N, Lee HJ, Lee JS, Patel S, Lee SJ (2002) Golgi fragmentation occurs in the cells with prefibrillar alpha-synuclein aggregates and precedes the formation of fibrillar inclusion. J Biol Chem 277:48984-48992.

Higo T, Hamada K, Hisatsune C, Nukina N, Hashikawa T, Hattori M, Nakamura T, Mikoshiba K (2010) Mechanism of ER stress-induced brain damage by IP(3) receptor. Neuron 68:865-878.

Hoozemans JJ, van Haastert ES, Eikelenboom P, de Vos RA, Rozemuller JM, Scheper W (2007) Activation of the unfolded protein response in Parkinson's disease. Biochem Biophys Res Commun 354:707-711.

Jiang Z, Belforte JE, Lu Y, Yabe Y, Pickel J, Smith CB, Je HS, Lu B, Nakazawa K (2010) eIF2alpha Phosphorylation-dependent translation in CA1 pyramidal cells impairs hippocampal memory consolidation without affecting general translation. J Neurosci 30:2582-2594.

Kim I, Xu W, Reed JC (2008) Cell death and endoplasmic reticulum stress: disease relevance and therapeutic opportunities. Nat Rev Drug Discov 7:1013-1030.

Kirik D, Rosenblad C, Burger C, Lundberg C, Johansen TE, Muzyczka N, Mandel RJ, Björklund A (2002) Parkinson-like neurodegeneration induced by targeted overexpression of alpha-synuclein in the nigrostriatal system. J Neurosci 22:2780-2791.

Koprich JB, Johnston TH, Reyes MG, Sun X, Brotchie JM (2010) Expression of human A53T alpha-synuclein in the rat substantia nigra using a novel $A A V 1 / 2$ vector produces a rapidly evolving pathology with protein aggregation, dystrophic neurite architecture and nigrostriatal degeneration with potential to model the pathology of Parkinson's disease. Mol Neurodegener 5:43.

Kozutsumi Y, Segal M, Normington K, Gething MJ, Sambrook J (1988) The presence of malfolded proteins in the endoplasmic reticulum signals the induction of glucose-regulated proteins. Nature 332:462-464.

Lee HJ, Patel S, Lee SJ (2005) Intravesicular localization and exocytosis of alpha-synuclein and its aggregates. J Neurosci 25:6016-6024.

Lee MK, Stirling W, Xu Y, Xu X, Qui D, Mandir AS, Dawson TM, Copeland NG, Jenkins NA, Price DL (2002) Human alpha-synuclein-harboring familial Parkinson's disease-linked Ala-53 $\rightarrow$ Thr mutation causes neurodegenerative disease with alpha-synuclein aggregation in transgenic mice. Proc Natl Acad Sci U S A 99:8968-8973.

Li W, Lee MK (2005) Antiapoptotic property of human alpha-synuclein in neuronal cell lines is associated with the inhibition of caspase- 3 but not caspase-9 activity. J Neurochem 93:1542-1550.

Li W, Hoffman PN, Stirling W, Price DL, Lee MK (2004a) Axonal transport of human alpha-synuclein slows with aging but is not affected by familial Parkinson's disease-linked mutations. J Neurochem 88:401-410.

Li W, Lesuisse C, Xu Y, Troncoso JC, Price DL, Lee MK (2004b) Stabilization of alpha-synuclein protein with aging and familial Parkinson's disease-linked A53T mutation. J Neurosci 24:7400-7409.

Li W, West N, Colla E, Pletnikova O, Troncoso JC, Marsh L, Dawson TM, Jäkälä P, Hartmann T, Price DL, Lee MK (2005) Aggregation promoting C-terminal truncation of alpha-synuclein is a normal cellular process and is enhanced by the familial Parkinson's disease-linked mutations. Proc Natl Acad Sci U S A 102:2162-2167.

Lin W, Kunkler PE, Harding HP, Ron D, Kraig RP, Popko B (2008) Enhanced integrated stress response promotes myelinating oligodendrocyte survival in response to interferon-gamma. Am J Pathol 173:1508-1517.

Liu Y, Yoo MJ, Savonenko A, Stirling W, Price DL, Borchelt DR, Mamounas L, Lyons WE, Blue ME, Lee MK (2008) Amyloid pathology is associated with progressive monoaminergic neurodegeneration in a transgenic mouse model of Alzheimer's disease. J Neurosci 28:13805-13814.

Lo Bianco C, Ridet JL, Schneider BL, Deglon N, Aebischer P (2002) alphaSynucleinopathy and selective dopaminergic neuron loss in a rat lentiviral-based model of Parkinson's disease. Proc Natl Acad Sci U S A 99:10813-10818.

Martin LJ, Pan Y, Price AC, Sterling W, Copeland NG, Jenkins NA, Price DL, Lee MK (2006) Parkinson's disease alpha-synuclein transgenic mice develop neuronal mitochondrial degeneration and cell death. J Neurosci 26:41-50.

Martinez-Vicente M, Talloczy Z, Kaushik S, Massey AC, Mazzulli J, Mosharov EV, Hodara R, Fredenburg R, Wu DC, Follenzi A, Dauer W, Przedborski S, Ischiropoulos H, Lansbury PT, Sulzer D, Cuervo AM (2008) Dopaminemodified alpha-synuclein blocks chaperone-mediated autophagy. J Clin Invest 118:777-788.

Nakagomi S, Barsoum MJ, Bossy-Wetzel E, Sütterlin C, Malhotra V, Lipton SA (2008) A Golgi fragmentation pathway in neurodegeneration. Neurobiol Dis 29:221-231.

Narayanan V, Scarlata S (2001) Membrane binding and self-association of alpha-synucleins. Biochemistry 40:9927-9934.

Nawrocki ST, Carew JS, Dunner K Jr, Boise LH, Chiao PJ, Huang P, Abbruzzese JL, McConkey DJ (2005) Bortezomib inhibits PKR-like endoplasmic reticulum (ER) kinase and induces apoptosis via ER stress in human pancreatic cancer cells. Cancer Res 65:11510-11519.

Obeso JA, Rodriguez-Oroz MC, Goetz CG, Marin C, Kordower JH, Rodriguez M, Hirsch EC, Farrer M, Schapira AH, Halliday G (2010) Missing pieces in the Parkinson's disease puzzle. Nat Med 16:653-661.

Pletnikova O, West N, Lee MK, Rudow GL, Skolasky RL, Dawson TM, Marsh L, Troncoso JC (2005) Abeta deposition is associated with enhanced cortical alpha-synuclein lesions in Lewy body diseases. Neurobiol Aging 26:1183-1192.

Py BF, Boyce M, Yuan J (2009) A critical role of eEF-2K in mediating autophagy in response to multiple cellular stresses. Autophagy 5:393-396.

Ron D, Walter P (2007) Signal integration in the endoplasmic reticulum unfolded protein response. Nat Rev Mol Cell Biol 8:519-529.

Saxena S, Cabuy E, Caroni P (2009) A role for motoneuron subtype-selective ER stress in disease manifestations of FALS mice. Nat Neurosci 12:627636.

Schallert T, Fleming SM, Leasure JL, Tillerson JL, Bland ST (2000) CNS plasticity and assessment of forelimb sensorimotor outcome in unilateral 
rat models of stroke, cortical ablation, parkinsonism and spinal cord injury. Neuropharmacology 39:777-787.

Silva RM, Ries V, Oo TF, Yarygina O, Jackson-Lewis V, Ryu EJ, Lu PD, Marciniak SJ, Ron D, Przedborski S, Kholodilov N, Greene LA, Burke RE (2005) CHOP/GADD153 is a mediator of apoptotic death in substantia nigra dopamine neurons in an in vivo neurotoxin model of parkinsonism. J Neurochem 95:974-986.

Smith WW, Jiang H, Pei Z, Tanaka Y, Morita H, Sawa A, Dawson VL, Dawson TM, Ross CA (2005) Endoplasmic reticulum stress and mitochondrial cell death pathways mediate A53T mutant alpha-synuclein-induced toxicity. Hum Mol Genet 14:3801-3811.

Sokka AL, Putkonen N, Mudo G, Pryazhnikov E, Reijonen S, Khiroug L, Belluardo N, Lindholm D, Korhonen L (2007) Endoplasmic reticulum stress inhibition protects against excitotoxic neuronal injury in the rat brain. J Neurosci 27:901-908.

Tallóczy Z, Jiang W, Virgin HW 4th, Leib DA, Scheuner D, Kaufman RJ, Eskelinen EL, Levine B (2002) Regulation of starvation- and virusinduced autophagy by the eIF2alpha kinase signaling pathway. Proc Natl Acad Sci U S A 99:190-195.

Vijayvergiya C, Beal MF, Buck J, Manfredi G (2005) Mutant superoxide dismutase 1 forms aggregates in the brain mitochondrial matrix of amyotrophic lateral sclerosis mice. J Neurosci 25:2463-2470.

Vila M, Przedborski S (2004) Genetic clues to the pathogenesis of Parkinson's disease. Nat Med 10 [Suppl]:S58-S62.
Volles MJ, Lansbury PT Jr (2007) Relationships between the sequence of alpha-synuclein and its membrane affinity, fibrillization propensity, and yeast toxicity. J Mol Biol 366:1510-1522.

Volpicelli-Daley LA, Luk KC, Patel TP, Tanik SA, Riddle DM, Stieber A, Meaney DF, Trojanowski JQ, Lee VM (2011) Exogenous alphasynuclein fibrils induce Lewy body pathology leading to synaptic dysfunction and neuron death. Neuron 72:57-71.

Wang J, Martin E, Gonzales V, Borchelt DR, Lee MK (2008) Differential regulation of small heat shock proteins in transgenic mouse models of neurodegenerative diseases. Neurobiol Aging 29:586-597.

Wang L, Popko B, Roos RP (2011) The unfolded protein response in familial amyotrophic lateral sclerosis. Hum Mol Genet 20:1008-1015.

Winslow AR, Chen CW, Corrochano S, Acevedo-Arozena A, Gordon DE, Peden AA, Lichtenberg M, Menzies FM, Ravikumar B, Imarisio S, Brown S, O'Kane CJ, Rubinsztein DC (2010) alpha-Synuclein impairs macroautophagy: implications for Parkinson's disease. J Cell Biol 190:1023-1037.

Zhang K, Kaufman RJ (2006) The unfolded protein response: a stress signaling pathway critical for health and disease. Neurology 66: S102-109.

Zhu Y, Fenik P, Zhan G, Sanfillipo-Cohn B, Naidoo N, Veasey SC (2008) Eif-2a protects brainstem motoneurons in a murine model of sleep apnea. J Neurosci 28:2168-2178. 